\title{
High-Order Analytic Expansion of Disturbing Function for Doubly Averaged Circular Restricted Three-Body Problem
}

\author{
Takashi Ito \\ National Astronomical Observatory of Japan, Osawa 2-21-1, Mitaka, Tokyo 181-8588, Japan \\ Correspondence should be addressed to Takashi Ito; tito@cfca.nao.ac.jp
}

Received 3 June 2016; Revised 18 August 2016; Accepted 19 September 2016

Academic Editor: Elbaz I. Abouelmagd

Copyright ( 2016 Takashi Ito. This is an open access article distributed under the Creative Commons Attribution License, which permits unrestricted use, distribution, and reproduction in any medium, provided the original work is properly cited.

\begin{abstract}
Terms in the analytic expansion of the doubly averaged disturbing function for the circular restricted three-body problem using the Legendre polynomial are explicitly calculated up to the fourteenth order of semimajor axis ratio $(\alpha)$ between perturbed and perturbing bodies in the inner case $(\alpha<1)$, and up to the fifteenth order in the outer case $(\alpha>1)$. The expansion outcome is compared with results from numerical quadrature on an equipotential surface. Comparison with direct numerical integration of equations of motion is also presented. Overall, the high-order analytic expansion of the doubly averaged disturbing function yields a result that agrees well with the numerical quadrature and with the numerical integration. Local extremums of the doubly averaged disturbing function are quantitatively reproduced by the high-order analytic expansion even when $\alpha$ is large. Although the analytic expansion is not applicable in some circumstances such as when orbits of perturbed and perturbing bodies cross or when strong mean motion resonance is at work, our expansion result will be useful for analytically understanding the long-term dynamical behavior of perturbed bodies in circular restricted three-body systems.
\end{abstract}

\section{Introduction}

In the long tradition of celestial mechanics, the restricted three-body problem has occupied a fundamental role. In this problem, the mass of one of the three bodies is assumed to be small enough so that it does not affect the motion of the other two bodies. The restricted three-body problem is often considered on a rotating coordinate where central body and perturbing body are always located on the $x$-axis. See Szebehely [1] for more general characteristics of the restricted three-body problem.

Among many variants of the restricted three-body problem, its circular version called the circular restricted threebody problem (hereafter referred to as CR3BP) has been studied particularly well, and it makes a basis for understanding solar system dynamics and many other fields in celestial mechanics. In this system, the perturbing body lies on a circular orbit around central body. As is well known, the degree of freedom of CR3BP becomes unity and the system turns into integrable once we average the disturbing function of the system by mean anomalies of perturbed and perturbing bodies. Theories of the so-called classical Lidov-Kozai cycle have been developed based on the integrable characteristics of the doubly averaged CR3BP [2-4], where stationary points of argument of pericenter $g$ around $\pm \pi / 2$ appear when the vertical component of the angular momentum of perturbed body is smaller than a certain value. The present paper deals with the doubly averaged CR3BP.

In the classical theory of the Lidov-Kozai cycle, the doubly averaged disturbing function is expanded using the Legendre polynomials of even orders. Putting $\alpha=a / a^{\prime}$, where $a$ is the semimajor axis of perturbed body and $a^{\prime}$ is that of perturbing body, only the lowest-order terms up to $O\left(\alpha^{2}\right)$ are considered in many of the studies along this line (e.g., [5-9]); it is the quadruple-order approximation. Recent studies of the so-called eccentric Lidov-Kozai mechanism (e.g., $[10-13])$ that deal with the eccentric restricted threebody problem (ER3BP), where the orbit of perturbing body has a finite eccentricity, are based on the octupole-order approximation of disturbing function up to $O\left(\alpha^{3}\right)$. It is now getting better known that the inclusion of octupole terms in the disturbing function substantially changes the dynamical behavior of ER3BP. Even in CR3BP, the quadruple-order approximation is not accurate enough when $\alpha$ is large. To 
eliminate this shortcoming, in the early days, Kozai [3] expanded the doubly averaged disturbing function of the inner CR3BP up to $O\left(\alpha^{8}\right)$. More recently, Laskar and Boué [14] calculated analytic expansions of the general threebody disturbing function together with a practical method to compute the Hansen coefficients. Laskar and Boué [14] explicitly showed expressions of secular disturbing function up to $O\left(\alpha^{14}\right)$ for planar problems and up to $O\left(\alpha^{5}\right)$ for spatial problems.

In the present paper we will show specific expressions of the analytic expansion of the doubly averaged spatial disturbing function up to $\mathrm{O}\left(\alpha^{14}\right)$ for the inner CR3BP $(\alpha<1)$ and up to $O\left(\alpha^{\prime 15}\right)$ for the outer CR3BP $\left(\alpha^{\prime}=\alpha^{-1}<1\right)$ using the Legendre polynomials. As most readers are aware, very wide varieties of studies have been already done on the analytic expansion of the doubly averaged disturbing function of CR3BP. Compared with previous literature, the present paper intentionally aims to be rather expository. The major purpose of this paper is to explicitly show expressions of the high-order analytic expansion of the doubly averaged disturbing function for CR3BP so that readers with interest can consult the high-order terms without going through algebraic manipulation by themselves. We also aim at analytically reproducing local extremums that secular disturbing function of CR3BP intrinsically has, particularly when $\alpha$ or $\alpha^{\prime}$ is large. Thus, even on a very basic subject like this, we have felt it advisable to give more details than would otherwise be necessary.

In Section 2 we give a brief description of the disturbing function of the three-body problem that we consider in the present paper. Section 3 goes to double averaging and analytic expansion of the disturbing function for CR3BP: general procedure (Section 3.1) and specific forms (from Sections 3.2 to 3.8). In Section 4 we show a comparison between the results obtained by the analytic expansion and by numerical quadrature. We also carried out direct numerical integration of equations of motion for comparison and show its result in Section 5. Section 6 is devoted to summary and discussion.

For readers' convenience, before getting into the main sections let us quickly write down the basic equations of motion of the system that we deal with in the present paper. The differential equation that we will consider is the simple classical Newtonian equation of motion

$$
\frac{d^{2} \mathbf{r}}{d t^{2}}+\mu \frac{\mathbf{r}}{r^{3}}=\nabla R
$$

where $\mathbf{r}$ indicates the position vector of the perturbed body and $\mu$ relates to the central mass. The disturbing function that plays a central role in this paper is denoted as $R$. As for a literal definition of the doubly averaged disturbing function, particularly its direct part, we use the following one:

$$
\left\langle\langle R\rangle_{l^{\prime}}\right\rangle_{l}=\frac{\mu^{\prime}}{4 \pi^{2}} \iint_{0}^{2 \pi} \frac{d l d l^{\prime}}{\Delta},
$$

where $\mu^{\prime}$ is related to the mass of perturbing body, $l$ and $l^{\prime}$ denote mean anomaly of perturbed and perturbing bodies, respectively, and $\Delta$ is the osculating distance of the two bodies in space. Consult later sections for detailed definitions of the variables in the above equations. Needless to say, the considered system contains only three bodies.

\section{Disturbing Function}

In the present paper we categorize CR3BP in two cases: (i) the inner case where the orbit of the perturbed body is located inside that of a perturbing body $(\alpha<1)$, such as the Sunasteroid-Jupiter system, and (ii) the outer case where the orbit of the perturbed body is located outside that of a perturbing body $\left(\alpha^{\prime}=\alpha^{-1}<1\right)$ such as the Sun-Neptune-TNO system (TNO $=$ Trans-Neptunian Object). The coorbital case $(\alpha=1)$ is out of the scope of the present paper.

Following the long-term convention of celestial mechanics, in the present paper we express the disturbing function $R$ of CR3BP in relative coordinates where the origin is located on the primary body (Figure 1(a)). In this coordinate system, the disturbing function that describes the perturbation on the motion of an object with mass $m$ due to the motion of another mass $m^{\prime}$ has the following general form (e.g., [15, p. 228]):

$$
R=\frac{\mu^{\prime}}{\Delta}-\mu^{\prime} \frac{\mathbf{r} \cdot \mathbf{r}^{\prime}}{r^{\prime 3}},
$$

where $\mu^{\prime}=\mathscr{G} m^{\prime}$ with the gravitational constant $\mathscr{G}, \mathbf{r}$ is the position vector of the mass $m$ with respect to the central body, $\mathbf{r}^{\prime}$ is the position vector of the mass $m^{\prime}$ with respect to the central body, and $\Delta=\left|\mathbf{r}^{\prime}-\mathbf{r}\right|$. In what follows we will consider only the first term of the right-hand side of (3) which is often referred to as the direct part. The second term is called the indirect part. As is well known, the indirect part makes no contribution to long-term dynamics of the system because it vanishes after the double averaging procedure, unless nonnegligible mean motion resonances are at work and we cannot simply employ the double averaging procedure.

When designating $S$ as the angle between the vectors $\mathbf{r}$ and $\mathbf{r}^{\prime}$, it is also well known that $1 / \Delta$ on the right-hand side of (3) can be expanded using the Legendre polynomials $P_{j}$ as

$$
\frac{1}{\Delta}=\frac{1}{r^{\prime}} \sum_{j=0}^{\infty}\left(\frac{r}{r^{\prime}}\right)^{j} P_{j}(\cos S),
$$

when $r<r^{\prime}$ (i.e., the inner case), and

$$
\frac{1}{\Delta}=\frac{1}{r} \sum_{j=0}^{\infty}\left(\frac{r^{\prime}}{r}\right)^{j} P_{j}(\cos S),
$$

when $r>r^{\prime}$ (i.e., the outer case). Once again, it is well known that the terms of $j=0$ and $j=1$ in (4) and (5) do not contribute to secular motion of the bodies, as they will vanish or become constant after the double averaging procedure. Hence in the remaining part of this paper we will just consider terms with $j \geq 2$ in (4) and (5).

Readers find the expressions of the disturbing functions (3), (4), and (5) and their derivations in many textbooks such as Brouwer and Clemence [16], Danby [17], or Murray 


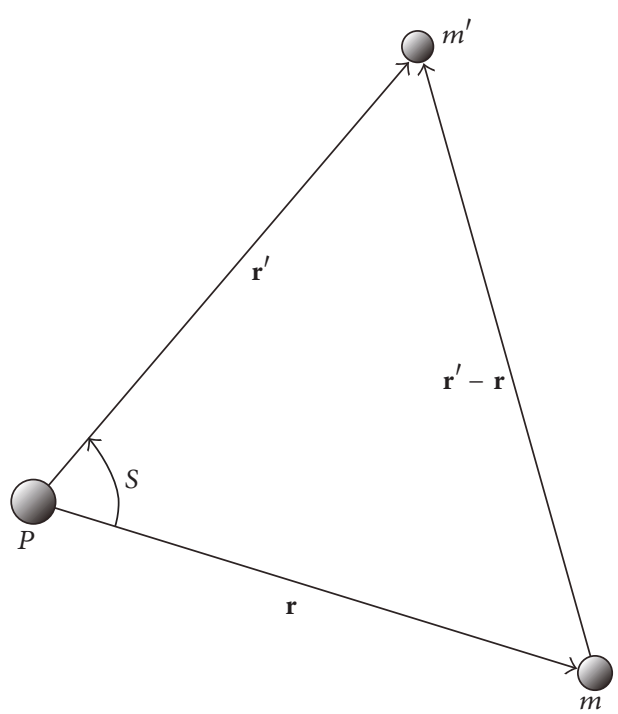

(a)

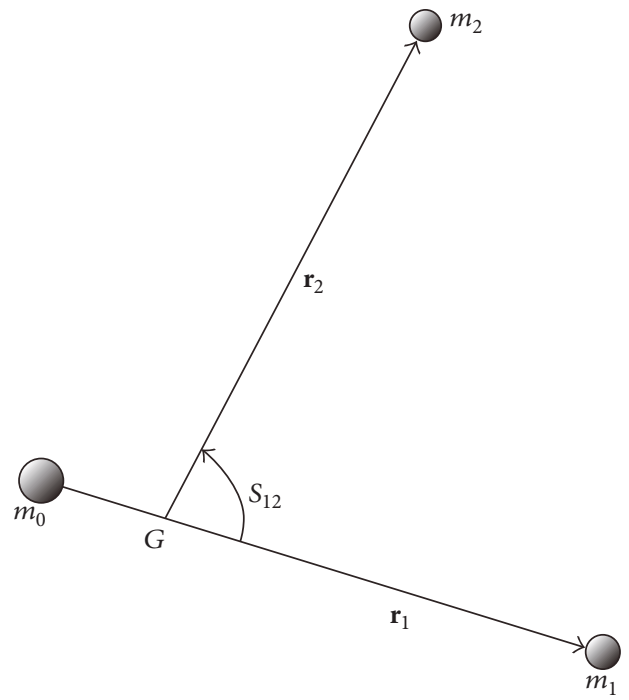

(b)

FIGURE 1: A schematic illustration of the three-body configuration considered here in two kinds of coordinate. (a) Relative coordinate. Both the vectors $\mathbf{r}$ and $\mathbf{r}^{\prime}$ originates from the central mass denoted as $P$. (b) Jacobi coordinate. The vector $\mathbf{r}_{1}$ is originated from the primary mass $\left(m_{0}\right)$, and the vector $\mathbf{r}_{2}$ is originated from the barycenter of the primary mass and the secondary mass $\left(m_{1}\right)$ denoted as $G$.

and Dermott [15]. In the inner case, the direct part of the disturbing function $\mu^{\prime} / \Delta$ can be derived also in a more general way. Consider a general three-body system with three masses: primary $m_{0}$, secondary $m_{1}$, and tertiary $m_{2}$. Now we use the Jacobi coordinate (e.g., [18]): measuring $m_{1}$ 's position vector $\mathbf{r}_{1}$ from $m_{0}$, measuring $m_{2}$ 's position vector $\mathbf{r}_{2}$ from the barycenter of $m_{0}$ and $m_{1}$, and $S_{12}$ is the angle between the vectors $\mathbf{r}_{1}$ and $\mathbf{r}_{2}$. Naturally $\mathbf{r}_{1}$ and $\mathbf{r}_{2}$ have different origins, and the angle $S_{12}$ is different from $S$ in general (see Figure 1(b)). We assume $r_{1}<r_{2}$. In this coordinate system, the equations of motion of $m_{1}$ and $m_{2}$ become (see $[16,19]$, for detailed derivation)

$$
\begin{aligned}
& \widetilde{m}_{1} \frac{d^{2} \mathbf{r}_{1}}{d t^{2}}=\frac{\partial F}{\partial \mathbf{r}_{1}}, \\
& \widetilde{m}_{2} \frac{d^{2} \mathbf{r}_{2}}{d t^{2}}=\frac{\partial F}{\partial \mathbf{r}_{2}},
\end{aligned}
$$

where

$$
\begin{aligned}
& \widetilde{m}_{1}=\frac{m_{0} m_{1}}{m_{0}+m_{1}}, \\
& \widetilde{m}_{2}=\frac{\left(m_{0}+m_{1}\right) m_{2}}{m_{0}+m_{1}+m_{2}}
\end{aligned}
$$

are reduced masses used in the Jacobi coordinate system (e.g., $[20,21])$, and $F$ is the common force function

$$
\begin{aligned}
F & =\mathscr{G}\left[\frac{m_{0} m_{1}}{r_{1}}+\frac{\left(m_{0}+m_{1}\right) m_{2}}{r_{2}}\right. \\
& \left.+\frac{1}{r_{2}} \sum_{j=2}^{\infty} M_{j}\left(\frac{r_{1}}{r_{2}}\right)^{j} P_{j}\left(\cos S_{12}\right)\right],
\end{aligned}
$$

where

$$
M_{j}=\frac{m_{0} m_{1} m_{2}\left(m_{0}^{j-1}-\left(-m_{1}\right)^{j-1}\right)}{\left(m_{0}+m_{1}\right)^{j}}
$$

is the mass factor. Using the force function $F$, this system can be written in a canonical form governed by a Hamiltonian. By expressing $a_{1}$ and $a_{2}$ as the semimajor axis of the orbits of the secondary and the tertiary (e.g., [22-25]), the Hamiltonian $\mathscr{H}$ becomes

$$
\begin{aligned}
\mathscr{H}= & \frac{\mathscr{S} m_{0} m_{1}}{2 a_{1}}+\frac{\mathscr{G}\left(m_{0}+m_{1}\right) m_{2}}{2 a_{2}} \\
& +\frac{\mathscr{G}}{r_{2}} \sum_{j=2}^{\infty} M_{j}\left(\frac{r_{1}}{r_{2}}\right)^{j} P_{j}\left(\cos S_{12}\right) .
\end{aligned}
$$

The first and the second terms of $\mathscr{H}$ in (10) drive the Keplerian motion of the secondary and tertiary mass, respectively. Note that the third term which represents the mutual interaction of the secondary and the tertiary does not include terms of $j=0$ or $j=1$. This is a typical consequence of the use of the Jacobi coordinate that separates the motions of three bodies into two separate binaries and their interactions by a single infinite series.

Now, let us think about the limit where $m_{1}$ is infinitesimally small; this would correspond to the inner R3BP. In this case, we divide the force function $F$ in (8) by $\widetilde{m}_{1}$ in (7) for normalization by mass before taking the limit. Then the third term of $F$ becomes

$$
\frac{F_{3 \mathrm{rd}}}{\widetilde{m}_{1}}=\frac{\mathscr{G} m_{2}}{r_{2}} \sum_{j=2}^{\infty} \frac{m_{0}^{j-1}-\left(-m_{1}\right)^{j-1}}{\left(m_{0}+m_{1}\right)^{j-1}}\left(\frac{r_{1}}{r_{2}}\right)^{j} P_{j}\left(\cos S_{12}\right) \text {. }
$$


Now we take the limit of $m_{1} \rightarrow 0$, and the position of $G$ in Figure 1(b) gets overlapped with the position of $m_{0}$. Thus we can replace $\mathbf{r}_{1}$ for $\mathbf{r}, \mathbf{r}_{2}$ for $\mathbf{r}^{\prime}, S_{12}$ for $S$, and $m_{2}$ for $m^{\prime}$ and will end up with an expression equivalent to the direct part of the disturbing function $R$ of the inner case written in the relative coordinate (4).

On the other hand, deriving the form of the disturbing function of the outer case written in the relative coordinate (5) by taking the mass-less limit $\left(m_{2} \rightarrow 0\right)$ of Hamiltonian (10) is difficult, if not impossible. In the outer case, the origin of the position vector of the tertiary $\left(\mathbf{r}_{2}\right)$ is the barycenter of the primary and secondary ( $G$ in Figure $1(b))$. But $G$ would not be overlapped with the position of the primary regardless of the value of the tertiary's mass $m_{2}$, unless $m_{1} \rightarrow 0$. Therefore, in the present paper, we will not mention the conversion of the general three-body Hamiltonian into the outer disturbing function written in the relative coordinate. In modern celestial mechanics, more and more methods for expanding disturbing function without using the relative coordinate are becoming available (e.g., $[14,26,27])$.

\section{Doubly Averaged Disturbing Function for CR3BP}

3.1. General Form. From (3) and (4), the direct part of the disturbing function for the inner CR3BP where $r^{\prime}=a^{\prime}$ becomes as follows:

$$
R=\frac{\mu^{\prime}}{a^{\prime}} \sum_{n=1}^{\infty}\left(\frac{r}{a^{\prime}}\right)^{2 n} P_{2 n}(\cos S),
$$

where we ignore the term of $n=0$. In (12) we also ignore all the terms including the odd Legendre polynomials $\left(P_{1}, P_{3}, P_{5}, P_{7}, P_{9}, \ldots\right)$ because they all vanish after the averaging procedure using mean anomaly of the perturbing body. Note that in the remaining part of this paper we will not consider the indirect part of the disturbing function either, as they do not have any secular dynamical contributions in nonresonant systems. Therefore we just use the variable $R$ for representing the entire part of the disturbing function.

Assuming there is no major resonant relationship between the mean motions of perturbed and perturbing bodies, we now try to get the double average of $R$ (12) over mean anomalies of both the bodies. Nonexistence of a resonant relationship means that the mean anomalies of perturbed and perturbing bodies (referred to as $l$ and $l^{\prime}$ in what follows) are independent from each other. The procedure to carry out double averaging of $R$ is straightforward as follows: Let us pick up the $n$th term of $R$ and name it as $R_{2 n}$. We have

$$
R_{2 n}=\frac{\mu^{\prime}}{a^{\prime}}\left(\frac{r}{a^{\prime}}\right)^{2 n} P_{2 n}(\cos S)
$$

First we average $R_{2 n}$ by mean anomaly of the perturbing body $l^{\prime}$, as

$$
\left\langle R_{2 n}\right\rangle_{l^{\prime}}=\frac{\mu^{\prime}}{a^{\prime}}\left(\frac{r}{a^{\prime}}\right)^{2 n}\left\langle P_{2 n}\right\rangle_{l^{\prime}}
$$

where

$$
\left\langle P_{2 n}\right\rangle_{l^{\prime}}=\frac{1}{2 \pi} \int_{0}^{2 \pi} P_{2 n}(\cos S) d l^{\prime} .
$$

The angle $S$ is expressed by orbital angles through a relationship (e.g., [3, Eq. (7) in p. 592])

$$
\begin{aligned}
\cos S= & \cos (f+g) \cos \left(f^{\prime}+g^{\prime}\right) \\
& +\cos i \sin (f+g) \sin \left(f^{\prime}+g^{\prime}\right),
\end{aligned}
$$

where $f, f^{\prime}$ are true anomalies of the perturbed and perturbing bodies, $g, g^{\prime}$ are arguments of pericenter of the perturbed and perturbing bodies, and $i$ is their mutual inclination measured at the node of the two orbits. We choose the orbital plane of the perturbing body as a reference plane for the entire system, and then $g$ and $g^{\prime}$ can be measured from the mutual node. Note that $g^{\prime}$ is not actually defined in CR3BP. Therefore, in (16) we regard $f^{\prime}+g^{\prime}$ as a single, fast-moving variable when we carry out averaging of (15). Practically, we can simply replace $\int d l^{\prime}$ for $\int d f^{\prime}$ in the discussion here.

To obtain $\left\langle P_{2 n}\right\rangle_{l^{\prime}}$ of (15), we calculate the average of $\cos ^{2 n} S$ by $l^{\prime}$ as

$$
\left\langle\cos ^{2 n} S\right\rangle_{l^{\prime}}=\frac{1}{2 \pi} \int_{0}^{2 \pi} \cos ^{2 n} S d l^{\prime}
$$

Then we average $\left\langle R_{2 n}\right\rangle_{l^{\prime}}$ of (14) by mean anomaly of the perturbed body $l$, as

$$
\left\langle\left\langle R_{2 n}\right\rangle_{l^{\prime}}\right\rangle_{l}=\frac{\mu^{\prime}}{a^{\prime}}\left(\frac{a}{a^{\prime}}\right)^{2 n} \frac{1}{2 \pi} \int_{0}^{2 \pi}\left(\frac{r}{a}\right)^{2 n}\left\langle P_{2 n}\right\rangle_{l^{\prime}} d l .
$$

If we switch the integration variable from $l$ to eccentric anomaly $u$, (18) becomes

$$
\begin{aligned}
& \left\langle\left\langle R_{2 n}\right\rangle_{l^{\prime}}\right\rangle_{l} \\
& =\frac{\mu^{\prime}}{a^{\prime}}\left(\frac{a}{a^{\prime}}\right)^{2 n} \frac{1}{2 \pi} \int_{0}^{2 \pi}(1-e \cos u)^{2 n+1}\left\langle P_{2 n}\right\rangle_{l^{\prime}} d u .
\end{aligned}
$$

We can obtain the doubly averaged disturbing function for the outer CR3BP in the same way as above. From (3) and (5), the direct part of the disturbing function $R^{\prime}$ for the outer CR3BP becomes as follows:

$$
R^{\prime}=\frac{\mu^{\prime}}{r} \sum_{n=1}^{\infty}\left(\frac{a^{\prime}}{r}\right)^{2 n} P_{2 n}(\cos S) .
$$

Note that our definition of $1 / \Delta$ for the outer case (5), hence also in (20), may be different from what is seen in conventional textbooks (e.g., [15, Eq. (6.22) in p. 229]): Roles of the dashed quantities $\left(r^{\prime}, \mu^{\prime}\right)$ may be the opposite. This difference comes from the fact that conventional textbooks always assume $r / r^{\prime}<1$, while we assume $r / r^{\prime}>1$ for the outer problem. This is because we make it a rule to always use dash (I) for the quantities of perturbing body, whether it is located inside or outside the perturbed body.

Similar to the procedures that we went through for the inner CR3BP, we again assume that there is no major 
resonant relationship between mean motions of perturbed and perturbing bodies in the outer CR3BP. We then try to get the double average of $R^{\prime}$ over mean anomalies of both the bodies. Let us pick the $n$th term of $R^{\prime}$ in (20) and name it $R_{2 n}^{\prime}$. We have

$$
R_{2 n}^{\prime}=\frac{\mu^{\prime}}{r}\left(\frac{a^{\prime}}{r}\right)^{2 n} P_{2 n}(\cos S) .
$$

First we average $R_{2 n}^{\prime}$ by mean anomaly of the perturbing body $l^{\prime}$, as

$$
\left\langle R_{2 n}^{\prime}\right\rangle_{l^{\prime}}=\frac{\mu^{\prime}}{r}\left(\frac{a^{\prime}}{r}\right)^{2 n}\left\langle P_{2 n}\right\rangle_{l^{\prime}},
$$

where $\left\langle P_{2 n}\right\rangle_{l^{\prime}}$ is already defined in (15).

Then we average $\left\langle R_{2 n}^{\prime}\right\rangle_{l^{\prime}}$ in (22) by mean anomaly of the perturbed body $l$, as

$$
\begin{aligned}
& \left\langle\left\langle R_{2 n}^{\prime}\right\rangle_{l^{\prime}}\right\rangle_{l} \\
& =\frac{\mu^{\prime}}{a^{\prime}}\left(\frac{a^{\prime}}{a}\right)^{2 n+1} \frac{1}{2 \pi} \int_{0}^{2 \pi}\left(\frac{a}{r}\right)^{2 n+1}\left\langle P_{2 n}\right\rangle_{l^{\prime}} d l .
\end{aligned}
$$

If we switch the integration variable from $l$ to true anomaly $f$, (23) becomes

$$
\begin{aligned}
\left\langle\left\langle R_{2 n}^{\prime}\right\rangle_{l^{\prime}}\right\rangle_{l}= & \frac{\mu^{\prime}}{a^{\prime}}\left(\frac{a^{\prime}}{a}\right)^{2 n+1} \frac{\left(1-e^{2}\right)^{-2 n+1 / 2}}{2 \pi} \\
& \cdot \int_{0}^{2 \pi}(1+e \cos f)^{2 n-1}\left\langle P_{2 n}\right\rangle_{l^{\prime}} d f
\end{aligned}
$$

Note that $\left\langle\left\langle R_{2 n}^{\prime}\right\rangle_{l^{\prime}}\right\rangle_{l}$ has the order of $O\left(\alpha^{\prime 2 n+1}\right)$ as in (23) and (24), not $O\left(\alpha^{\prime 2 n}\right)$.

In Sections 3.2 to 3.8 we show the expressions of $\left\langle\cos ^{2 n} S\right\rangle_{l^{\prime}}$ in (17), $\left\langle P_{2 n}\right\rangle_{l^{\prime}}$ in (15), the Legendre polynomial $P_{2 n}(x)$ in its original form, $\left\langle\left\langle R_{2 n}\right\rangle_{l^{\prime}}\right\rangle_{l}$ in (19), and $\left\langle\left\langle R_{2 n}^{\prime}\right\rangle_{l^{\prime}}\right\rangle_{l}$ in (24) for $2 n=$ $2,4,6,8,10,12,14$. We used Maple ${ }^{\mathrm{TM}}$ for algebraic manipulation to obtain the series of expressions. Note that in what follows we use $\sin i$ instead of cos $i$ because it generally makes the formulas simpler. For this reason, some of the expressions look apparently different from what was presented in the previous literature in spite of their equivalence.

3.2. $2 n=2$. At this order, the corresponding component of the disturbing function for the inner problem $R_{2}$ is of $O\left(\alpha^{2}\right)$, and that for the outer problem $R_{2}^{\prime}$ is of $O\left(\alpha^{\prime 3}\right)$.

We just describe the resulting expressions of the expansion as follows: Let us emphasize again that the dashed quantities such as $\mu^{\prime}$ and $a^{\prime}$ are those of the perturbing body, whether its orbit is located inside or outside of the orbit of the perturbed body.

$$
\begin{aligned}
& \left\langle\cos ^{2} S\right\rangle_{l^{\prime}}=\frac{1}{4} \sin ^{2} i \cos 2(f+g)-\frac{1}{4} \sin ^{2} i+\frac{1}{2}, \\
& P_{2}(x)=\frac{3}{2} x^{2}-\frac{1}{2}, \\
& \left\langle P_{2}\right\rangle_{l^{\prime}}=\frac{3}{8} \sin ^{2} i \cos 2(f+g)-\frac{3}{8} \sin ^{2} i+\frac{1}{4}, \\
& \left\langle\left\langle R_{2}\right\rangle_{l^{\prime}}\right\rangle_{l}=\frac{\mu^{\prime}}{a^{\prime}}\left(\frac{a}{a^{\prime}}\right)^{2} \\
& \cdot\left[\frac{15}{16} e^{2} \sin ^{2} i \cos 2 g-\frac{1}{16}\left(3 e^{2}+2\right)\left(3 \sin ^{2} i-2\right)\right], \\
& \left\langle\left\langle R_{2}^{\prime}\right\rangle_{l^{\prime}}\right\rangle_{l}=\frac{\mu^{\prime}}{a^{\prime}}\left(\frac{a^{\prime}}{a}\right)^{3}\left(1-e^{2}\right)^{-3 / 2}\left[-\frac{3}{8} \sin ^{2} i+\frac{1}{4}\right] .
\end{aligned}
$$

The expression in (28) shows the leading term of the doubly averaged disturbing function that causes the classical (circular) Lidov-Kozai cycle in the inner CR3BP that we have often seen in the previous literature. Meanwhile, the expression in (29) shows the leading term of the doubly averaged disturbing function for the outer CR3BP, but somehow we do not see it often. We should note that the leading term of the doubly averaged disturbing function for the outer problem (29) does not contain dependence on $g$. The $g$-dependence in the doubly averaged outer CR3BP first shows up in the next order: $2 n=4$. This is why the Lidov-Kozai mechanism for the outer CR3BP is more subtle than the inner one, particularly when $\alpha^{\prime}$ is small, and perhaps this is why we rarely see the expression in the literature.

3.3. $2 n=4$. At this order, the corresponding component of the disturbing function for the inner problem $R_{4}$ is of $O\left(\alpha^{4}\right)$, and that for the outer problem $R_{4}^{\prime}$ is of $O\left(\alpha^{\prime 5}\right)$.

$$
\begin{aligned}
& \left\langle\cos ^{4} S\right\rangle_{l^{\prime}}=\frac{3}{64} \sin ^{4} i \cos 4(f+g)-\frac{3}{16} \sin ^{2} i\left(\sin ^{2} i\right. \\
& -2) \cos 2(f+g)+\frac{9}{64} \sin ^{4} i-\frac{3}{8} \sin ^{2} i+\frac{3}{8}, \\
& P_{4}(x)=\frac{35}{8} x^{4}-\frac{15}{4} x^{2}+\frac{3}{8}, \\
& \left\langle P_{4}\right\rangle_{l^{\prime}}=\frac{105}{512} \sin ^{4} i \cos 4(f+g)-\frac{15}{128} \sin ^{2} i\left(7 \sin ^{2} i\right. \\
& -6) \cos 2(f+g)+\frac{315}{512} \sin ^{4} i-\frac{45}{64} \sin ^{2} i+\frac{9}{64}, \\
& \left\langle\left\langle R_{4}\right\rangle_{l^{\prime}}\right\rangle_{l}=\frac{\mu^{\prime}}{a^{\prime}}\left(\frac{a}{a^{\prime}}\right)^{4}\left[\frac{6615}{4096} e^{4} \sin ^{4} i \cos 4 g-\frac{315}{1024} e^{2}\right. \\
& \cdot \sin ^{2} i\left(e^{2}+2\right)\left(7 \sin ^{2} i-6\right) \cos 2 g \\
& +\frac{9}{4096}\left(15 e^{4}+40 e^{2}+8\right) \\
& \left..\left(35 \sin ^{4} i+40 \sin ^{2} i+8\right)\right],
\end{aligned}
$$




$$
\begin{aligned}
& \left\langle\left\langle R_{4}^{\prime}\right\rangle_{l^{\prime}}\right\rangle_{l}=\frac{\mu^{\prime}}{a^{\prime}}\left(\frac{a^{\prime}}{a}\right)^{5}\left(1-e^{2}\right)^{-7 / 2}\left[-\frac{45}{512} e^{2} \sin ^{2} i\right. \\
& \cdot\left(7 \sin ^{2} i-6\right) \cos 2 g+\frac{9}{1024}\left(3 e^{2}+2\right) \\
& \left.\cdot\left(35 \sin ^{4} i-40 \sin ^{2} i+8\right)\right] .
\end{aligned}
$$

Note that we now see the $g$-dependence of the doubly averaged disturbing function for the outer CR3BP in the expression of (34).

3.4. $2 n=6$. At this order, the corresponding component of the disturbing function for the inner problem $R_{6}$ is of $O\left(\alpha^{6}\right)$, and that for the outer problem $R_{6}^{\prime}$ is of $O\left(\alpha^{17}\right)$.

$$
\begin{aligned}
& \left\langle\cos ^{6} S\right\rangle_{l^{\prime}}=\frac{5}{512} \sin ^{6} i \cos 6(f+g)-\frac{15}{256} \sin ^{4} i\left(\sin ^{2} i\right. \\
& -2) \cos 4(f+g)+\frac{15}{512} \sin ^{2} i\left(5 \sin ^{4} i-16 \sin ^{2} i\right. \\
& +16) \cos 2(f+g)-\frac{5}{256}\left(\sin ^{2} i-2\right)\left(5 \sin ^{4} i-8\right. \\
& \left.\cdot \sin ^{2} i+8\right) \\
& P_{6}(x)=\frac{231}{16} x^{6}-\frac{315}{16} x^{4}+\frac{105}{16} x^{2}-\frac{5}{16}, \\
& \left\langle P_{6}\right\rangle_{l^{\prime}}=\frac{1155}{8192} \sin ^{6} i \cos 6(f+g)-\frac{315}{4096} \sin ^{4} i(11 \\
& \left.\cdot \sin ^{2} i-10\right) \cos 4(f+g)+\frac{525}{8192} \sin ^{2} i\left(33 \sin ^{4} i\right. \\
& \left.-48 \sin ^{2} i+16\right) \cos 2(f+g)-\frac{5775}{4096} \sin ^{6} i+\frac{4725}{2048} \\
& \cdot \sin ^{4} i-\frac{525}{512} \sin ^{2} i+\frac{25}{256} \\
& \left\langle\left\langle R_{6}\right\rangle_{l^{\prime}}\right\rangle_{l}=\frac{\mu^{\prime}}{a^{\prime}}\left(\frac{a}{a^{\prime}}\right)^{6}\left[\frac{495495}{131072} e^{6} \sin ^{6} i \cos 6 g\right. \\
& -\frac{10395}{65536} e^{4} \sin ^{4} i\left(3 e^{2}+10\right)\left(11 \sin ^{2} i-10\right) \cos 4 g \\
& +\frac{1575}{131072} e^{2} \sin ^{2} i\left(15 e^{4}+80 e^{2}+48\right) \\
& \text { - }\left(33 \sin ^{4} i-48 \sin ^{2} i+16\right) \cos 2 g \\
& -\frac{25}{65536}\left(35 e^{6}+210 e^{4}+168 e^{2}+16\right) \\
& \text {. } \left.\left(231 \sin ^{6} i-378 \sin ^{4} i+168 \sin ^{2} i-16\right)\right] \text {, } \\
& \left\langle\left\langle R_{6}^{\prime}\right\rangle_{l^{\prime}}\right\rangle_{l}=\frac{\mu^{\prime}}{a^{\prime}}\left(\frac{a^{\prime}}{a}\right)^{7}\left(1-e^{2}\right)^{-11 / 2}\left[-\frac{1575}{65536} e^{4} \sin ^{4} i\right. \\
& \cdot\left(11 \sin ^{2} i-10\right) \cos 4 g+\frac{2625}{32768} e^{2} \sin ^{2} i\left(e^{2}+2\right)
\end{aligned}
$$

3.5. $2 n=8$. At this order, the corresponding component of the disturbing function for the inner problem $R_{8}$ is of $O\left(\alpha^{8}\right)$, and that for the outer problem $R_{8}^{\prime}$ is of $O\left(\alpha^{\prime 9}\right)$.

$$
\begin{aligned}
& \left\langle\cos ^{8} S\right\rangle_{l^{\prime}}=\frac{35}{16384} \sin ^{8} i \cos 8(f+g)-\frac{35}{2048} \sin ^{6} i \\
& \cdot\left(\sin ^{2} i-2\right) \cos 6(f+g)+\frac{35}{4096} \sin ^{4} i\left(7 \sin ^{4} i\right. \\
& \left.-24 \sin ^{2} i+24\right) \cos 4(f+g)-\frac{35}{2048} \sin ^{2} i\left(\sin ^{2} i\right. \\
& \text {-2) }\left(7 \sin ^{4} i-16 \sin ^{2} i+16\right) \cos 2(f+g) \\
& +\frac{1225}{16384} \sin ^{8} i-\frac{175}{512} \sin ^{6} i+\frac{315}{512} \sin ^{4} i-\frac{35}{64} \sin ^{2} i \\
& +\frac{35}{128} \\
& P_{8}(x)=\frac{6435}{128} x^{8}-\frac{3003}{32} x^{6}+\frac{3465}{64} x^{4}-\frac{315}{32} x^{2} \\
& +\frac{35}{128} \\
& \left\langle P_{8}\right\rangle_{l^{\prime}}=\frac{225225}{2097152} \sin ^{8} i \cos 8(f+g)-\frac{15015}{262144} \sin ^{6} i \\
& \cdot\left(15 \sin ^{2} i-14\right) \cos 6(f+g)+\frac{24255}{524288} \sin ^{4} i(65 \\
& \left.\cdot \sin ^{4} i-104 \sin ^{2} i+40\right) \cos 4(f+g)-\frac{11025}{262144} \\
& \text { - } \sin ^{2} i\left(143 \sin ^{6} i-286 \sin ^{4} i+176 \sin ^{2} i-32\right) \\
& \cdot \cos 2(f+g)+\frac{7882875}{2097152} \sin ^{8} i-\frac{525525}{65536} \sin ^{6} i \\
& +\frac{363825}{65536} \sin ^{4} i-\frac{11025}{8192} \sin ^{2} i+\frac{1225}{16384}, \\
& \left\langle\left\langle R_{8}\right\rangle_{l^{\prime}}\right\rangle_{l}=\frac{\mu^{\prime}}{a^{\prime}}\left(\frac{a}{a^{\prime}}\right)^{8}\left[\frac{2737609875}{268435456} e^{8} \sin ^{8} i \cos 8 g\right. \\
& -\frac{10735725}{33554432} e^{6} \sin ^{6} i\left(3 e^{2}+14\right)\left(15 \sin ^{2} i-14\right) \\
& \cdot \cos 6 g+\frac{17342325}{67108864} e^{4} \sin ^{4} i\left(e^{4}+8 e^{2}+8\right)\left(65 \sin ^{4} i\right. \\
& \left.-104 \sin ^{2} i+40\right) \cos 4 g-\frac{606375}{33554432} e^{2} \sin ^{2} i\left(7 e^{6}\right. \\
& \left.+70 e^{4}+112 e^{2}+32\right)\left(143 \sin ^{6} i-286 \sin ^{4} i\right.
\end{aligned}
$$




$$
\begin{aligned}
& \left.+176 \sin ^{2} i-32\right) \cos 2 g+\frac{1225}{268435456}\left(315 e^{8}\right. \\
& \left.+3360 e^{6}+6048 e^{4}+2304 e^{2}+128\right)\left(6435 \sin ^{8} i\right. \\
& \left.\left.-13728 \sin ^{6} i+9504 \sin ^{4} i-2304 \sin ^{2} i+128\right)\right], \\
& \left\langle\left\langle R_{8}^{\prime}\right\rangle_{l^{\prime}}\right\rangle_{l}=\frac{\mu^{\prime}}{a^{\prime}}\left(\frac{a^{\prime}}{a}\right)^{9}\left(1-e^{2}\right)^{-15 / 2}\left[-\frac{105105}{16777216} e^{6}\right. \\
& \cdot \sin ^{6} i\left(15 \sin ^{2} i-14\right) \cos 6 g+\frac{169785}{16777216} e^{4} \sin ^{4} i \\
& \cdot\left(3 e^{2}+10\right)\left(65 \sin ^{4} i-104 \sin ^{2} i+40\right) \cos 4 g \\
& -\frac{77175}{16777216} e^{2} \sin ^{2} i\left(15 e^{4}+80 e^{2}+48\right)\left(143 \sin ^{6} i\right. \\
& \left.-286 \sin ^{4} i+176 \sin ^{2} i-32\right) \cos 2 g \\
& +\frac{1225}{33554432}\left(35 e^{6}+210 e^{4}+168 e^{2}+16\right) \\
& \cdot\left(6435 \sin ^{8} i-13728 \sin ^{6} i+9504 \sin ^{4} i\right. \\
& \left.\left.-2304 \sin ^{2} i+128\right)\right] \cdot
\end{aligned}
$$

3.6. $2 n=10$. At this order, the corresponding component of the disturbing function for the inner problem $R_{10}$ is of $O\left(\alpha^{10}\right)$, and that for the outer problem $R_{10}^{\prime}$ is of $O\left(\alpha^{\prime 11}\right)$.

$$
\begin{aligned}
& \left\langle\cos ^{10} S\right\rangle_{l^{\prime}}=\frac{63}{131072} \sin ^{10} i \cos 10(f+g)-\frac{315}{65536} \\
& \cdot \sin ^{8} i\left(\sin ^{2} i-2\right) \cos 8(f+g)+\frac{315}{131072} \sin ^{6} i(9 \\
& \left.\cdot \sin ^{4} i-32 \sin ^{2} i+32\right) \cos 6(f+g)-\frac{315}{16384} \sin ^{4} i \\
& \cdot\left(\sin ^{2} i-2\right)\left(3 \sin ^{4} i-8 \sin ^{2} i+8\right) \cos 4(f+g) \\
& +\frac{315}{65536} \sin ^{2} i\left(21 \sin ^{8} i-112 \sin ^{6} i+240 \sin ^{4} i\right. \\
& \left.-256 \sin ^{2} i+128\right) \cos 2(f+g)-\frac{63}{65536}\left(\sin ^{2} i\right. \\
& -2)\left(63 \sin ^{8} i-224 \sin ^{6} i+352 \sin ^{4} i-256 \sin ^{2} i\right. \\
& +128), \\
& P_{10}(x)=\frac{46189}{256} x^{10}-\frac{109395}{256} x^{8}+\frac{45045}{128} x^{6} \\
& -\frac{15015}{128} x^{4}+\frac{3465}{256} x^{2}-\frac{63}{256}, \\
& \left\langle P_{10}\right\rangle_{l^{\prime}}=\frac{2909907}{33554432} \sin ^{10} i \cos 10(f+g) \\
& -\frac{765765}{16777216} \sin ^{8} i\left(19 \sin ^{2} i-18\right) \cos 8(f+g)
\end{aligned}
$$

$$
\begin{gathered}
\left\langle\left\langle R_{10}^{\prime}\right\rangle_{l^{\prime}}\right\rangle_{l}=\frac{\mu^{\prime}}{a^{\prime}}\left(\frac{a^{\prime}}{a}\right)^{11}\left(1-e^{2}\right)^{-19 / 2} \\
\cdot\left[-\frac{6891885}{4294967296} e^{8} \sin ^{8} i\left(19 \sin ^{2} i-18\right) \cos 8 g\right. \\
+\frac{1216215}{1073741824} e^{6} \sin ^{6} i\left(3 e^{2}+14\right)\left(323 \sin ^{4} i\right.
\end{gathered}
$$

$\cdot \cos 6(f+g)-\frac{135135}{4194304} \sin ^{4} i\left(323 \sin ^{6} i-714\right.$

- $\left.\sin ^{4} i+504 \sin ^{2} i-112\right) \cos 4(f+g)$

$+\frac{72765}{16777216} \sin ^{2} i\left(4199 \sin ^{8} i-10608 \sin ^{6} i+9360\right.$

$\left.\cdot \sin ^{4} i-3328 \sin ^{2} i+384\right) \cos 2(f+g)$

$-\frac{183324141}{16777216} \sin ^{10} i+\frac{241215975}{8388608} \sin ^{8} i$

$-\frac{14189175}{524288} \sin ^{6} i+\frac{2837835}{262144} \sin ^{4} i-\frac{218295}{131072} \sin ^{2} i$

$+\frac{3969}{65536}$

$\left\langle\left\langle R_{10}\right\rangle_{l^{\prime}}\right\rangle_{l}=\frac{\mu^{\prime}}{a^{\prime}}\left(\frac{a}{a^{\prime}}\right)^{10}\left[\frac{256592689353}{8589934592} e^{10} \sin ^{10} i\right.$

$\cdot \cos 10 g-\frac{9646341705}{4294967296} e^{8} \sin ^{8} i\left(e^{2}+6\right)\left(19 \sin ^{2} i\right.$

$-18) \cos 8 g+\frac{89594505}{8589934592} e^{6} \sin ^{6} i\left(15 e^{4}+160 e^{2}\right.$

+224) $\left(323 \sin ^{4} i-544 \sin ^{2} i+224\right) \cos 6 g$

$-\frac{36891855}{1073741824} e^{4} \sin ^{4} i\left(5 e^{6}+70 e^{4}+168 e^{2}+80\right)$

- $\left(323 \sin ^{6} i-714 \sin ^{4} i+504 \sin ^{2} i-112\right) \cos 4 g$

$+\frac{2837835}{4294967296} e^{2} \sin ^{2} i\left(21 e^{8}+336 e^{6}+1008 e^{4}\right.$

$\left.+768 e^{2}+128\right)\left(4199 \sin ^{8} i-10608 \sin ^{6} i\right.$

$\left.+9360 \sin ^{4} i-3328 \sin ^{2} i+384\right) \cos 2 g$

$-\frac{3969}{4294967296}\left(693 e^{10}+11550 e^{8}+36960 e^{6}\right.$

$\left.+31680 e^{4}+7040 e^{2}+256\right)\left(46189 \sin ^{10} i\right.$

$-121550 \sin ^{8} i+114400 \sin ^{6} i-45760 \sin ^{4} i$

$\left.\left.+7040 \sin ^{2} i-256\right)\right]$ 


$$
\begin{aligned}
& \left.-544 \sin ^{2} i+224\right) \cos 6 g-\frac{8513505}{268435456} e^{4} \sin ^{4} i\left(e^{4}\right. \\
& \left.+8 e^{2}+8\right)\left(323 \sin ^{6} i-714 \sin ^{4} i+504 \sin ^{2} i\right. \\
& -112) \cos 4 g+\frac{654885}{536870912} e^{2} \sin ^{2} i\left(7 e^{6}+70 e^{4}\right. \\
& \left.+112 e^{2}+32\right)\left(4199 \sin ^{8} i-10608 \sin ^{6} i\right. \\
& \left.+9360 \sin ^{4} i-3328 \sin ^{2} i+384\right) \cos 2 g \\
& -\frac{3969}{2147483648}\left(315 e^{8}+3360 e^{6}+6048 e^{4}\right. \\
& \left.+2304 e^{2}+128\right)\left(46189 \sin ^{10} i-121550 \sin ^{8} i\right. \\
& +114400 \sin ^{6} i-45760 \sin ^{4} i+7040 \sin ^{2} i \\
& -256)]
\end{aligned}
$$

3.7. $2 n=12$. At this order, the corresponding component of the disturbing function for the inner problem $R_{12}$ is of $O\left(\alpha^{12}\right)$, and that for the outer problem $R_{12}^{\prime}$ is of $O\left(\alpha^{\prime 13}\right)$.

$$
\begin{aligned}
& \left\langle\cos ^{12} S\right\rangle_{l^{\prime}}=\frac{231}{2097152} \sin ^{12} i \cos 12(f+g)-\frac{693}{524288} \\
& \cdot \sin ^{10} i\left(\sin ^{2} i-2\right) \cos 10(f+g)+\frac{693}{1048576} \sin ^{8} i \\
& \cdot\left(11 \sin ^{4} i-40 \sin ^{2} i+40\right) \cos 8(f+g)-\frac{1155}{524288} \\
& \text { - } \sin ^{6} i\left(\sin ^{2} i-2\right)\left(11 \sin ^{4} i-32 \sin ^{2} i+32\right) \cos 6(f \\
& +g)+\frac{3465}{2097152} \sin ^{4} i\left(33 \sin ^{8} i-192 \sin ^{6} i+448\right. \\
& \left.\cdot \sin ^{4} i-512 \sin ^{2} i+256\right) \cos 4(f+g)-\frac{693}{262144} \\
& \text { - } \sin ^{2} i\left(\sin ^{2} i-2\right)\left(33 \sin ^{8} i-144 \sin ^{6} i+272 \sin ^{4} i\right. \\
& \left.-256 \sin ^{2} i+128\right) \cos 2(f+g)+\frac{53361}{1048576} \sin ^{12} i \\
& -\frac{43659}{131072} \sin ^{10} i+\frac{121275}{131072} \sin ^{8} i-\frac{5775}{4096} \sin ^{6} i \\
& +\frac{10395}{8192} \sin ^{4} i-\frac{693}{1024} \sin ^{2} i+\frac{231}{1024}, \\
& P_{12}(x)=\frac{676039}{1024} x^{12}-\frac{969969}{512} x^{10}+\frac{2078505}{1024} x^{8} \\
& -\frac{255255}{256} x^{6}+\frac{225225}{1024} x^{4}-\frac{9009}{512} x^{2}+\frac{231}{1024}, \\
& \left\langle P_{12}\right\rangle_{l^{\prime}}=\frac{156165009}{2147483648} \sin ^{12} i \cos 12(f+g) \\
& -\frac{20369349}{536870912} \sin ^{10} i\left(23 \sin ^{2} i-22\right) \cos 10(f+g)
\end{aligned}
$$

$$
\begin{aligned}
& +\frac{32008977}{1073741824} \sin ^{8} i\left(161 \sin ^{4} i-280 \sin ^{2} i+120\right) \\
& \cdot \cos 8(f+g)-\frac{2807805}{536870912} \sin ^{6} i\left(3059 \sin ^{6} i\right. \\
& \left.-7182 \sin ^{4} i+5472 \sin ^{2} i-1344\right) \cos 6(f+g) \\
& +\frac{10405395}{2147483648} \sin ^{4} i\left(7429 \sin ^{8} i-20672 \sin ^{6} i\right. \\
& \left.+20672 \sin ^{4} i-8704 \sin ^{2} i+1280\right) \cos 4(f+g) \\
& -\frac{2081079}{268435456} \sin ^{2} i\left(7429 \sin ^{10} i-22610 \sin ^{8} i\right. \\
& \left.+25840 \sin ^{6} i-13600 \sin ^{4} i+3200 \sin ^{2} i-256\right) \\
& \cdot \cos 2(f+g)+\frac{36074117079}{1073741824} \sin ^{12} i \\
& -\frac{14115958857}{134217728} \sin ^{10} i+\frac{16804712925}{134217728} \sin ^{8} i \\
& -\frac{294819525}{4194304} \sin ^{6} i+\frac{156080925}{8388608} \sin ^{4} i-\frac{2081079}{1048576} \\
& \sin ^{2} i+\frac{53361}{1048576},
\end{aligned}
$$$$
\left\langle\left\langle R_{12}\right\rangle_{l^{\prime}}\right\rangle_{l}=\frac{\mu^{\prime}}{a^{\prime}}\left(\frac{a}{a^{\prime}}\right)^{12}\left[\frac{203026224075675}{2199023255552} e^{12} \sin ^{12} i\right.
$$$$
\cdot \cos 12 g-\frac{1059267256047}{549755813888} e^{10} \sin ^{10} i\left(3 e^{2}+22\right)
$$$$
\cdot\left(23 \sin ^{2} i-22\right) \cos 10 g+\frac{361861484985}{1099511627776} e^{8} \sin ^{8} i
$$$$
\cdot\left(3 e^{4}+40 e^{2}+72\right)\left(161 \sin ^{4} i-280 \sin ^{2} i+120\right)
$$$$
\cdot \cos 8 g-\frac{6348447105}{549755813888} e^{6} \sin ^{6} i\left(5 e^{6}+90 e^{4}\right.
$$$$
\left.+288 e^{2}+192\right)\left(3059 \sin ^{6} i-7182 \sin ^{4} i\right.
$$$$
\left.+5472 \sin ^{2} i-1344\right) \cos 6 g+\frac{1238242005}{2199023255552} e^{4}
$$$$
\cdot \sin ^{4} i\left(45 e^{8}+960 e^{6}+4032 e^{4}+4608 e^{2}+1280\right)
$$$$
\text { . }\left(7429 \sin ^{8} i-20672 \sin ^{6} i+20672 \sin ^{4} i\right.
$$$$
\left.-8704 \sin ^{2} i+1280\right) \cos 4 g-\frac{72837765}{274877906944} e^{2}
$$$$
\cdot \sin ^{2} i\left(99 e^{10}+2310 e^{8}+11088 e^{6}+15840 e^{4}\right.
$$$$
\left.+7040 e^{2}+768\right)\left(7429 \sin ^{10} i-22610 \sin ^{8} i\right.
$$$$
\left.+25840 \sin ^{6} i-13600 \sin ^{4} i+3200 \sin ^{2} i-256\right)
$$$$
\cdot \cos 2 g+\frac{53361}{1099511627776}\left(3003 e^{12}+72072 e^{10}\right.
$$$$
+360360 e^{8}+549120 e^{6}+274560 e^{4}+39936 e^{2}
$$ 


$$
\begin{aligned}
& +1024)\left(676039 \sin ^{12} i-2116296 \sin ^{10} i\right. \\
& +2519400 \sin ^{8} i-1414400 \sin ^{6} i+374400 \sin ^{4} i \\
& \left.\left.-39936 \sin ^{2} i+1024\right)\right] \\
& \left\langle\left\langle R_{12}^{\prime}\right\rangle_{l^{\prime}}\right\rangle_{l}=\frac{\mu^{\prime}}{a^{\prime}}\left(\frac{a^{\prime}}{a}\right)^{13}\left(1-e^{2}\right)^{-23 / 2} \\
& \text {. }\left[-\frac{224062839}{549755813888} e^{10} \sin ^{10} i\left(23 \sin ^{2} i-22\right) \cos 10 g\right. \\
& +\frac{1760493735}{549755813888} e^{8} \sin ^{8} i\left(e^{2}+6\right)\left(161 \sin ^{4} i\right. \\
& \left.-280 \sin ^{2} i+120\right) \cos 8 g-\frac{92657565}{549755813888} e^{6} \sin ^{6} i \\
& \cdot\left(15 e^{4}+160 e^{2}+224\right)\left(3059 \sin ^{6} i-7182 \sin ^{4} i\right. \\
& \left.+5472 \sin ^{2} i-1344\right) \cos 6 g+\frac{343378035}{274877906944} e^{4} \\
& \cdot \sin ^{4} i\left(5 e^{6}+70 e^{4}+168 e^{2}+80\right)\left(7429 \sin ^{8} i\right. \\
& \left.-20672 \sin ^{6} i+20672 \sin ^{4} i-8704 \sin ^{2} i+1280\right) \\
& \cdot \cos 4 g-\frac{114459345}{137438953472} e^{2} \sin ^{2} i\left(21 e^{8}+336 e^{6}\right. \\
& \left.+1008 e^{4}+768 e^{2}+128\right)\left(7429 \sin ^{10} i\right. \\
& -22610 \sin ^{8} i+25840 \sin ^{6} i-13600 \sin ^{4} i \\
& \left.+3200 \sin ^{2} i-256\right) \cos 2 g \\
& +\frac{53361}{274877906944}\left(693 e^{10}+11550 e^{8}+36960 e^{6}\right. \\
& \left.+31680 e^{4}+7040 e^{2}+256\right)\left(676039 \sin ^{12} i\right. \\
& -2116296 \sin ^{10} i+2519400 \sin ^{8} i-1414400 \sin ^{6} i \\
& \left.\left.+374400 \sin ^{4} i-39936 \sin ^{2} i+1024\right)\right] \text {. }
\end{aligned}
$$

3.8. $2 n=14$. At this order, the corresponding component of the disturbing function for the inner problem $R_{14}$ is of $O\left(\alpha^{14}\right)$, and that for the outer problem $R_{14}^{\prime}$ is of $O\left(\alpha^{\prime 15}\right)$.

$$
\begin{aligned}
& \left\langle\cos ^{14} S\right\rangle_{l^{\prime}}=\frac{429}{16777216} \sin ^{14} i \cos 14(f+g) \\
& -\frac{3003}{8388608} \sin ^{12} i\left(\sin ^{2} i-2\right) \cos 12(f+g) \\
& +\frac{3003}{16777216} \sin ^{10} i\left(13 \sin ^{4} i-48 \sin ^{2} i+48\right) \\
& \cdot \cos 10(f+g)-\frac{3003}{4194304} \sin ^{8} i\left(\sin ^{2} i-2\right)(13
\end{aligned}
$$

$\left.\cdot \sin ^{4} i-40 \sin ^{2} i+40\right) \cos 8(f+g)+\frac{3003}{16777216}$

$\cdot \sin ^{6} i\left(143 \sin ^{8} i-880 \sin ^{6} i+2160 \sin ^{4} i-2560\right.$

$\left.\cdot \sin ^{2} i+1280\right) \cos 6(f+g)-\frac{3003}{8388608} \sin ^{4} i\left(\sin ^{2} i\right.$

- 2) $\left(143 \sin ^{8} i-704 \sin ^{6} i+1472 \sin ^{4} i-1536\right.$

$\left.\cdot \sin ^{2} i+768\right) \cos 4(f+g)+\frac{3003}{16777216} \sin ^{2} i(429$

$\cdot \sin ^{12} i-3168 \sin ^{10} i+10080 \sin ^{8} i-17920 \sin ^{6} i$

$\left.+19200 \sin ^{4} i-12288 \sin ^{2} i+4096\right) \cos 2(f+g)$

$-\frac{429}{4194304}\left(\sin ^{2} i-2\right)\left(429 \sin ^{12} i-2376 \sin ^{10} i\right.$

$+5832 \sin ^{8} i-7936 \sin ^{6} i+6528 \sin ^{4} i-3072 \sin ^{2} i$

$+1024)$

$$
\begin{aligned}
& P_{14}(x)=\frac{5014575}{2048} x^{14}-\frac{16900975}{2048} x^{12}+\frac{22309287}{2048} \\
& \cdot x^{10}-\frac{14549535}{2048} x^{8}+\frac{4849845}{2048} x^{6}-\frac{765765}{2048} x^{4} \\
& +\frac{45045}{2048} x^{2}-\frac{429}{2048}
\end{aligned}
$$$$
\left\langle P_{14}\right\rangle_{l^{\prime}}=\frac{2151252675}{34359738368} \sin ^{14} i \cos 14(f+g)
$$$$
-\frac{557732175}{17179869184} \sin ^{12} i\left(27 \sin ^{2} i-26\right) \cos 12(f+g)
$$$$
+\frac{870062193}{34359738368} \sin ^{10} i\left(45 \sin ^{2} i-44\right)\left(5 \sin ^{2} i-4\right)
$$$$
\cdot \cos 10(f+g)-\frac{189143955}{8589934592} \sin ^{8} i\left(1035 \sin ^{6} i\right.
$$$$
\left.-2530 \sin ^{4} i+2024 \sin ^{2} i-528\right) \cos 8(f+g)
$$$$
+\frac{693527835}{34359738368} \sin ^{6} i\left(3105 \sin ^{8} i-9200 \sin ^{6} i\right.
$$$$
\left.+9936 \sin ^{4} i-4608 \sin ^{2} i+768\right) \cos 6(f+g)
$$$$
-\frac{328513185}{17179869184} \sin ^{4} i\left(6555 \sin ^{10} i-21850 \sin ^{8} i\right.
$$$$
+27968 \sin ^{6} i-17024 \sin ^{4} i+4864 \sin ^{2} i-512 \text { ) }
$$$$
\cdot \cos 4(f+g)+\frac{19324305}{34359738368} \sin ^{2} i\left(334305 \sin ^{12} i\right.
$$

$-1188640 \sin ^{10} i+1664096 \sin ^{8} i-1157632 \sin ^{6} i$

$\left.+413440 \sin ^{4} i-69632 \sin ^{2} i+4096\right) \cos 2(f+g)$

$-\frac{922887397575}{8589934592} \sin ^{14} i+\frac{1674869721525}{4294967296} \sin ^{12} i$ 


$$
\begin{aligned}
& -\frac{602953099749}{1073741824} \sin ^{10} i+\frac{218461268025}{536870912} \sin ^{8} i \\
& -\frac{10402917525}{67108864} \sin ^{6} i+\frac{985539555}{33554432} \sin ^{4} i \\
& -\frac{19324305}{8388608} \sin ^{2} i+\frac{184041}{4194304} \\
& \left\langle\left\langle R_{14}\right\rangle_{l^{\prime}}\right\rangle_{l}=\frac{\mu^{\prime}}{a^{\prime}}\left(\frac{a}{a^{\prime}}\right)^{14}\left[\frac{20856061239960375}{70368744177664} e^{14}\right. \\
& \cdot \sin ^{14} i \cos 14 g-\frac{186452654763375}{35184372088832} e^{12} \sin ^{12} i\left(3 e^{2}\right. \\
& \text { +26) }\left(27 \sin ^{2} i-26\right) \cos 12 g+\frac{32318460158985}{70368744177664} \\
& \cdot e^{10} \sin ^{10} i\left(5 e^{4}+80 e^{2}+176\right)\left(45 \sin ^{2} i-44\right) \\
& \cdot\left(5 \sin ^{2} i-4\right) \cos 10 g-\frac{1405150441695}{17592186044416} e^{8} \sin ^{8} i \\
& \cdot\left(7 e^{6}+154 e^{4}+616 e^{2}+528\right)\left(1035 \sin ^{6} i\right. \\
& \left.-2530 \sin ^{4} i+2024 \sin ^{2} i-528\right) \cos 8 g \\
& +\frac{672028472115}{70368744177664} e^{6} \sin ^{6} i\left(21 e^{8}+560 e^{6}+3024 e^{4}\right. \\
& \left.+4608 e^{2}+1792\right)\left(3105 \sin ^{8} i-9200 \sin ^{6} i\right. \\
& \left.+9936 \sin ^{4} i-4608 \sin ^{2} i+768\right) \cos 6 g \\
& -\frac{318329276265}{35184372088832} e^{4} \sin ^{4} i\left(11 e^{10}+330 e^{8}\right. \\
& \left.+2112 e^{6}+4224 e^{4}+2816 e^{2}+512\right)\left(6555 \sin ^{10} i\right. \\
& -21850 \sin ^{8} i+27968 \sin ^{6} i-17024 \sin ^{4} i \\
& \left.+4864 \sin ^{2} i-512\right) \cos 4 g+\frac{328513185}{70368744177664} e^{2} \\
& \cdot \sin ^{2} i\left(429 e^{12}+13728 e^{10}+96096 e^{8}+219648 e^{6}\right. \\
& \left.+183040 e^{4}+53248 e^{2}+4096\right)\left(334305 \sin ^{12} i\right. \\
& -1188640 \sin ^{10} i+1664096 \sin ^{8} i-1157632 \sin ^{6} i \\
& \left.+413440 \sin ^{4} i-69632 \sin ^{2} i+4096\right) \cos 2 g \\
& -\frac{184041}{17592186044416}\left(6435 e^{14}+210210 e^{12}\right. \\
& +1513512 e^{10}+3603600 e^{8}+3203200 e^{6} \\
& \left.+1048320 e^{4}+107520 e^{2}+2048\right)\left(5014575 \sin ^{14} i\right. \\
& -18201050 \sin ^{12} i+26209512 \sin ^{10} i \\
& -18992400 \sin ^{8} i+7235200 \sin ^{6} i-1370880 \sin ^{4} i \\
& \left.\left.+107520 \sin ^{2} i-2048\right)\right] \text {, }
\end{aligned}
$$

$$
\begin{aligned}
& \left\langle\left\langle R_{14}^{\prime}\right\rangle_{l^{\prime}}\right\rangle_{l}=\frac{\mu^{\prime}}{a^{\prime}}\left(\frac{a^{\prime}}{a}\right)^{15}\left(1-e^{2}\right)^{-27 / 2} \\
& \cdot\left[-\frac{7250518275}{70368744177664} e^{12} \sin ^{12} i\left(27 \sin ^{2} i-26\right)\right. \\
& \cdot \cos 12 g+\frac{11310808509}{35184372088832} e^{10} \sin ^{10} i\left(3 e^{2}+22\right) \\
& \text {. }\left(45 \sin ^{2} i-44\right)\left(5 \sin ^{2} i-4\right) \cos 10 g \\
& -\frac{27047585565}{17592186044416} e^{8} \sin ^{8} i\left(3 e^{4}+40 e^{2}+72\right) \\
& \text {. }\left(1035 \sin ^{6} i-2530 \sin ^{4} i+2024 \sin ^{2} i-528\right) \\
& \cdot \cos 8 g+\frac{99174480405}{35184372088832} e^{6} \sin ^{6} i\left(5 e^{6}+90 e^{4}\right. \\
& \left.+288 e^{2}+192\right)\left(3105 \sin ^{8} i-9200 \sin ^{6} i\right. \\
& \left.+9936 \sin ^{4} i-4608 \sin ^{2} i+768\right) \cos 6 g \\
& -\frac{46977385455}{70368744177664} e^{4} \sin ^{4} i\left(45 e^{8}+960 e^{6}+4032 e^{4}\right. \\
& \left.+4608 e^{2}+1280\right)\left(6555 \sin ^{10} i-21850 \sin ^{8} i\right. \\
& \left.+27968 \sin ^{6} i-17024 \sin ^{4} i+4864 \sin ^{2} i-512\right) \\
& \cdot \cos 4 g+\frac{251215965}{17592186044416} e^{2} \sin ^{2} i\left(99 e^{10}+2310 e^{8}\right. \\
& \left.+11088 e^{6}+15840 e^{4}+7040 e^{2}+768\right) \\
& \text { - }\left(334305 \sin ^{12} i-1188640 \sin ^{10} i+1664096 \sin ^{8} i\right. \\
& -1157632 \sin ^{6} i+413440 \sin ^{4} i-69632 \sin ^{2} i \\
& +4096) \cos 2 g-\frac{184041}{8796093022208}\left(3003 e^{12}\right. \\
& +72072 e^{10}+360360 e^{8}+549120 e^{6}+274560 e^{4} \\
& \left.+39936 e^{2}+1024\right)\left(5014575 \sin ^{14} i\right. \\
& -18201050 \sin ^{12} i+26209512 \sin ^{10} i \\
& -18992400 \sin ^{8} i+7235200 \sin ^{6} i-1370880 \sin ^{4} i \\
& \left.\left.+107520 \sin ^{2} i-2048\right)\right] \text {. }
\end{aligned}
$$

\section{Comparison with Numerical Quadrature}

To graphically show the validity of the high-order analytic expansion of the doubly averaged disturbing function that we have presented, let us carry out numerical quadrature for comparison. The literal definition of the doubly averaged disturbing function, particularly its direct part, becomes from (3) 


$$
\left\langle\langle R\rangle_{l^{\prime}}\right\rangle_{l}=\frac{\mu^{\prime}}{4 \pi^{2}} \iint_{0}^{2 \pi} \frac{d l d l^{\prime}}{\Delta} .
$$

Since the orbit of the perturbing body is circular in CR3BP, we can turn the double integral of (52) into a single integral using the complete elliptic integral of the first kind (e.g., [28-30]). To compare the numerical quadrature result with that from the analytic expansion of disturbing function, we draw equipotential curves on the $(x, y)=(e \cos g, e \sin g)$ plane. Since $g$ is an angle that intrinsically rotates, use of this polar-type coordinate is reasonable; therefore, it has often been used in the previous literature (e.g., [31-33]). All the numerical quadratures including the calculation of elliptic integral presented in this section are achieved using the functions implemented in GNU Scientific Library (GSL; http://www.gnu.org/software/gsl/).

As mentioned, the doubly averaged CR3BP makes an integrable system with just one degree of freedom. Then the state of the system is determined by just two constant parameters: $\alpha$ (or $\alpha^{\prime}$ ) and (square of) normalized vertical component of the perturbed body's angular momentum $k^{2}$ defined as (e.g., [2, 34-37])

$$
k^{2}=\left(1-e^{2}\right) \cos ^{2} i
$$

We selected several combinations of $\left(\alpha, k^{2}\right)$ and drew equipotential curves using the analytically expanded doubly averaged disturbing function, as well as using the numerical quadrature (Figure 2). Note that from the definition of (53), the theoretical largest value of $e$ is achieved when $\cos i=1$ as $e_{\max }=\sqrt{1-k^{2}}$. Similarly, the theoretical smallest value of $\cos i$ is achieved when $e=0$ as $|\cos i|_{\min }=k$.

Figures 2(a) and 2(b) are for the inner case, and Figures 2(c)-2(f) are for the outer case. In each panel of Figure 2, the partial circle with red represents a line where the orbits of perturbed and perturbing bodies cross at the ascending node of the perturbed body. The partial circle with blue represents a line where the orbits of perturbed and perturbing bodies cross at the descending node of the perturbed body. These two circles are functions just of $a$ and $a^{\prime}$ (i.e., functions of $\alpha$ ), and the relationship between $a, e, g$, and $a^{\prime}$ is expressed as (e.g., $[37-40])$

$$
a^{\prime}=\frac{a\left(1-e^{2}\right)}{1 \pm e \cos g} .
$$

In the denominator of the right-hand term of (54), the positive sign takes place when the orbit intersect happens at the ascending node of perturbed body (when $f=-g$, where $f$ denotes perturbed body's true anomaly). The negative sign takes place when the encounter happens at the descending node of perturbed body (when $f=\pi-g$ ). Using the variables $x=e \cos g$ and $y=e \sin g$, (54) can be rewritten as

$$
\left(x \pm \frac{1}{2 \alpha}\right)^{2}+y^{2}=\left(1-\frac{1}{2 \alpha}\right)^{2}
$$

and it is more obvious that (55) represents a pair of circles on the $(x, y)$ plane.
Note that the orbit-crossing lines introduce singularities in $N$-body Hamiltonian (e.g., $[41,42]$ ), and $R$ (or $R^{\prime}$ ) is continuous but not regular across the orbit-crossing lines. Therefore the analytic expansion of the doubly averaged disturbing function is not applicable to the region very close to the orbit-crossing lines (e.g., [43]). Note also that each panel in Figure 2 has an individual contour interval, which represents a fixed interval of disturbing potential in each of the systems. Therefore, regions with dense contours imply that the potential gradient is steep there.

Since the high-order analytic expansion of disturbing function must have its significance when $\alpha$ (or $\alpha^{\prime}$ ) is large, we chose relatively large $\alpha$ (or $\alpha^{\prime}$ ) so that we cannot ignore their high-order powers. For example, when $2 n=14$ and $\alpha=0.50$, $\alpha^{14}=6.103515625 \times 10^{-5}$ which we may ignore. But when $2 n=14$ and $\alpha=0.90, \alpha^{14}=0.22876792454961$, which is not ignorable. In Figure 2(a) where $\left(\alpha, k^{2}\right)=(0.90,0.900)$, effect of the high-order analytic expansion is well exhibited. As is widely known, there is no stationary point for argument of pericenter $g$ while $k^{2}>0.6$ in the doubly averaged inner CR3BP at the quadruple-order approximation $(2 n=2)$. However when $\alpha$ becomes large, $g$ acquires stationary points at $g= \pm \pi / 2$ even when $k^{2}>0.6$. This is what we see in the quadrature result in the leftmost panel in Figure 2(a). Kozai [3] derived expressions of high-order analytic expansion of the doubly averaged disturbing function for the inner CR3BP up to $2 n=8$. But the stationary points of $g$ seen in the rightmost panel of Figure 2(a) (for quadrature) are not reproduced even by using the $2 n=8$ analytic terms. We confirmed that the stationary points at $g= \pm \pi / 2$ in this parameter set $\left(\alpha, k^{2}\right)=(0.90,0.900)$ first appear in the analytic approximation up to $2 n=12$, and the value of $e$ at the stationary points is better reproduced using the analytic expansion of $2 n=14$, as seen in Figure 2(a). Note that other sets of stationary points at $g=0, \pi$ seen in the quadrature result in the leftmost panel are not reproduced by the analytic expansion of disturbing function, since they are out of the orbit-crossing lines.

Note that we did not draw all the contours near the outer boundary $\left(e=e_{\max }\right)$ indicated by the dashed circles, particularly in the panels in the left three columns. This is mainly because the data size of the figure would be too large if we drew all the contours with a fixed interval of disturbing potential toward the outer boundary, and also because the contour intervals would become too narrow. Moreover, we believe that we can grab major topological patterns of disturbing potential in each panel even if we draw contours only partially, at least inside the orbit-crossing lines.

Figure 2(b) is for $\left(\alpha, k^{2}\right)=(0.90,0.400)$. They exemplify some limitations of the high-order analytic expansion of disturbing function that we have carried out. Although a saddle point seen in the quadrature result at the origin $(x, y)=(0,0)$ is reproduced in the results of the analytic expansion of disturbing function, we see spurious local extremums along the $y$-axis near the boundary of $e=e_{\max }$ in the $2 n=14$ case. The spurious local extremums are not so remarkable in the $2 n=8$ case (although they surely exist), but there seem to exist spurious saddle points around 

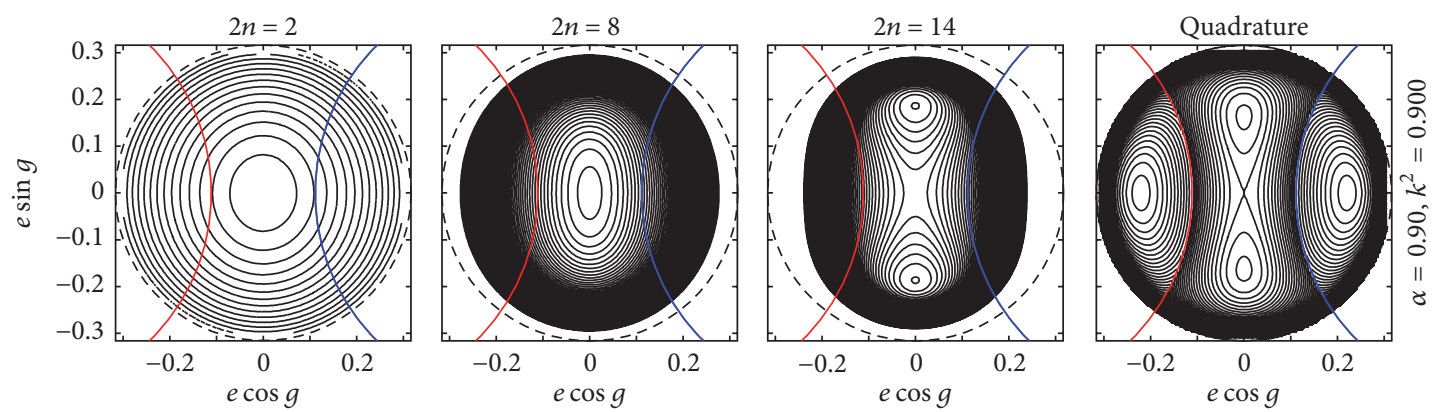

(a)
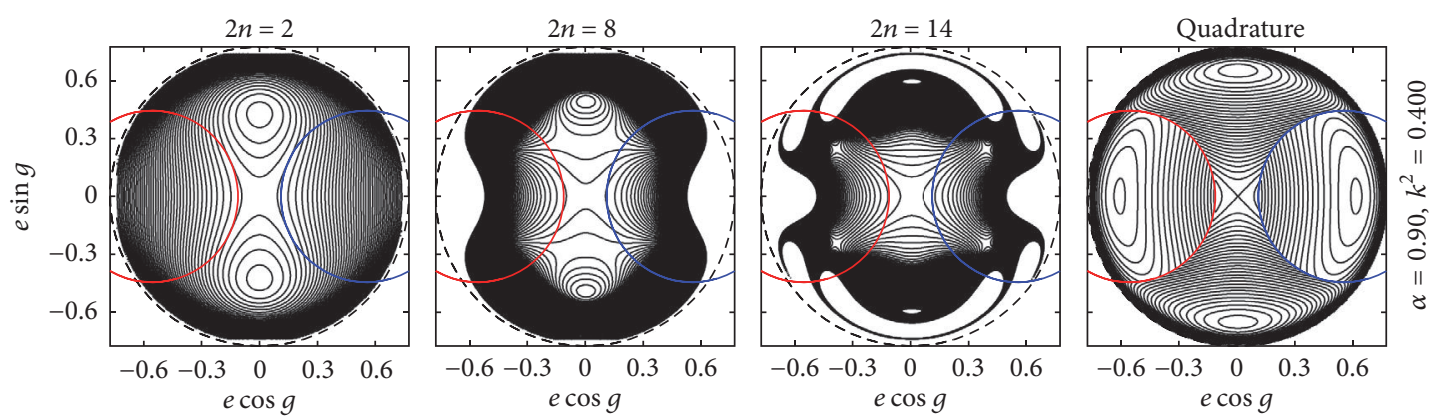

(b)
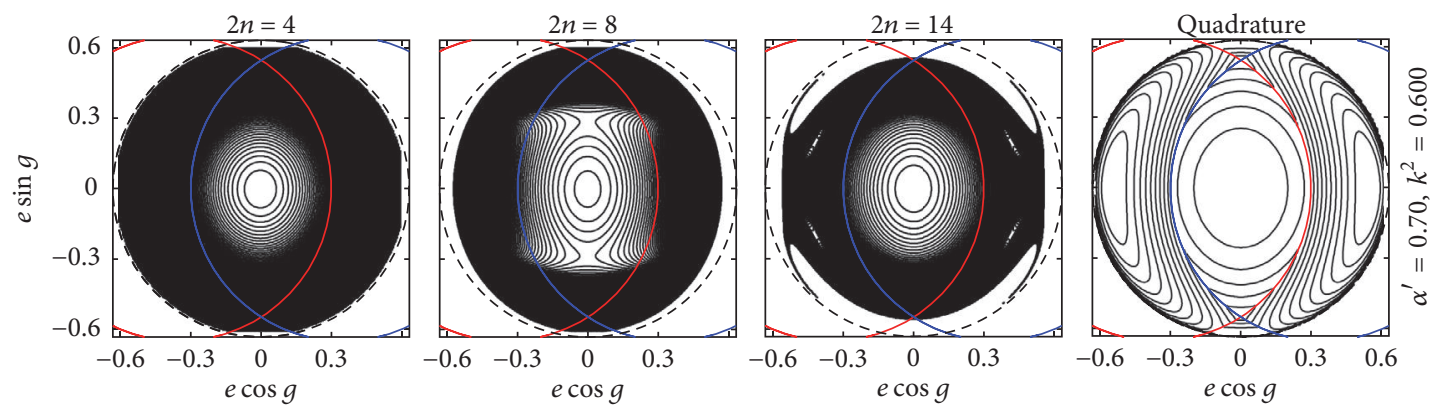

(c)
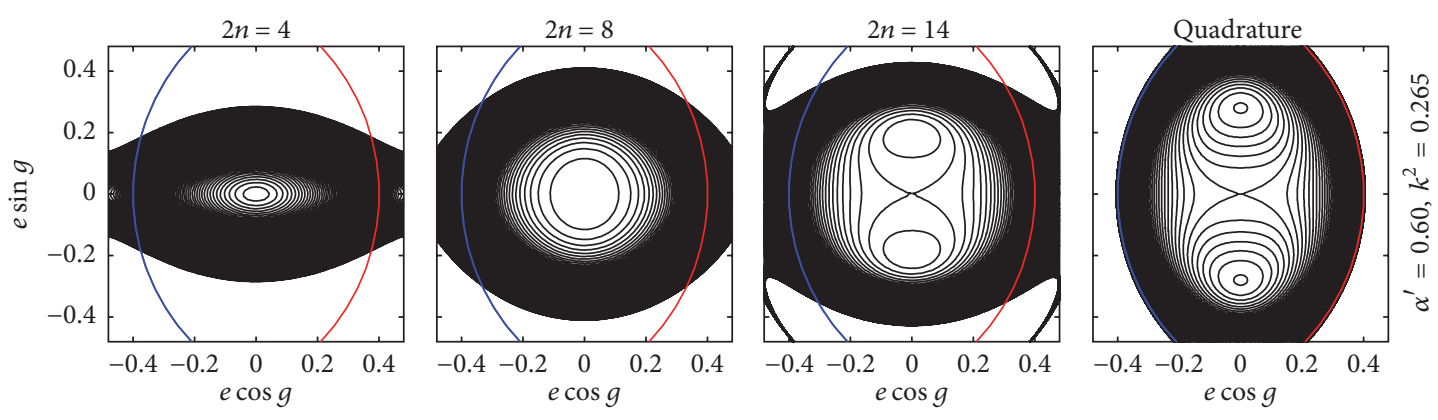

(d)
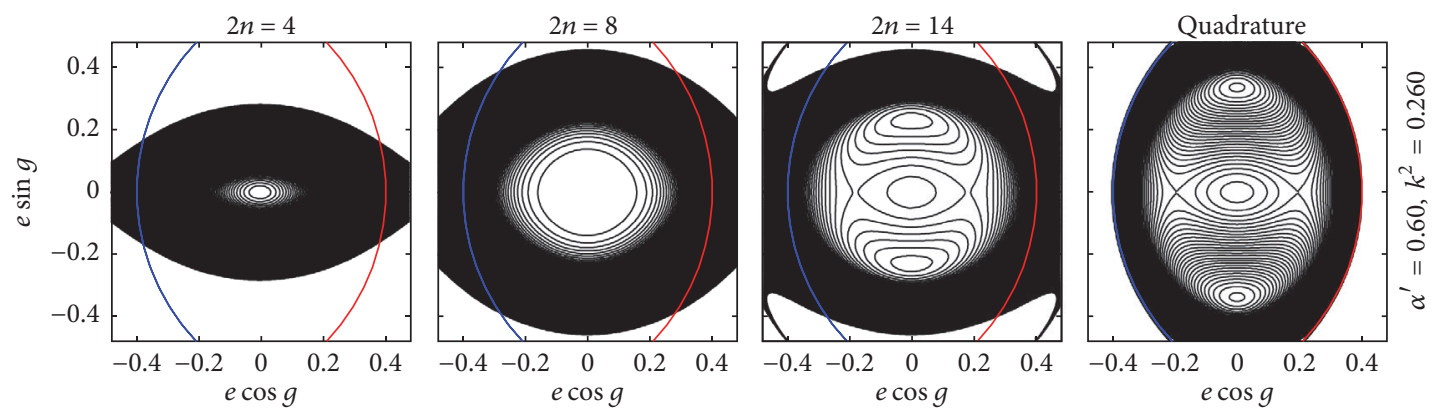

(e)

FIGURE 2: Continued. 

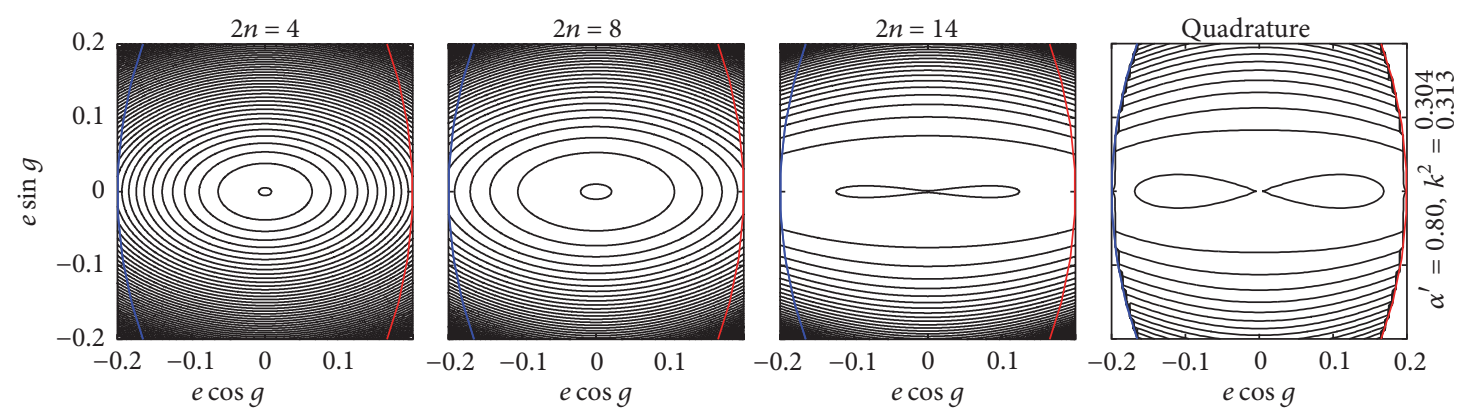

(f)

FIGURE 2: Equipotential curves computed through the doubly averaged disturbing function and plotted on the plane $(x, y)=(e$ cos $g, e$ sin $g)$. Columns are, from the left, $2 n=2$ (for (a), (b)) or $2 n=4$ (for (c), (d), (e), and (f)), $2 n=8,2 n=14$, and the numerical quadrature defined by (52). Rows are, from the top, (a) $\left(\alpha, k^{2}\right)=(0.90,0.900)$, (b) $\left(\alpha, k^{2}\right)=(0.90,0.400),(\mathrm{c})\left(\alpha^{\prime}, k^{2}\right)=(0.70,0.600),(\mathrm{d})\left(\alpha^{\prime}, k^{2}\right)=(0.60,0.265),(\mathrm{e})$ $\left(\alpha^{\prime}, k^{2}\right)=(0.60,0.260)$, and (f) $\left(\alpha^{\prime}, k^{2}\right)=(0.80,0.304)$ for the analytic expansion and $\left(\alpha^{\prime}, k^{2}\right)=(0.80,0.313)$ for the numerical quadrature. The black dashed circles represent the theoretical maximum eccentricity $\left(e_{\max }\right)$ of each of the systems. The red and the blue partial circles represent the conditions where the orbits of perturbed and perturbing bodies cross at the ascending node (red) and at the descending node (blue) of the perturbed body.

$y=e \sin g \sim \pm 0.3$ that do not appear in the quadrature result. Thus we say that the analytic expansion of the doubly averaged disturbing function for the inner CR3BP, at least up to these orders, is applicable to systems with moderate eccentricity when $\alpha$ is large. Note that it apparently seems that the leftmost panel for the analytic expansion of the order of $2 n=2$ looks the closest to the quadrature result. However, we think this is just a coincidence, because the $2 n=2$ expansion includes only the terms of $\cos 2 g$ as in (28), and $\left\langle\left\langle R_{2}\right\rangle_{l^{\prime}}\right\rangle_{l}$ would not be influenced by terms of $\cos 2 p g$ with $p \geq 2$.

Figure 2(c) is for the doubly averaged outer CR3BP when $\left(\alpha^{\prime}, k^{2}\right)=(0.70,0.600)$. In this case, the high-order analytic expansion of the doubly averaged disturbing function $(2 n=$ 14) reproduces the quadrature result inside the orbit-crossing lines. However, the medium-order expansion $(2 n=8)$ yields artificial saddle points along the $y$-axis. We confirmed that these spurious saddle points show up in the expansion of $2 n=10$ too (panels are not shown here). We thus say that the highest-order analytic expansion of $2 n=14$ pushes these saddle points out of the orbit-crossing lines, and the equipotential curves become similar to the quadrature result, at least inside the orbit-crossing lines where $e$ is moderate.

In Figure $2(\mathrm{~d})$ when $\left(\alpha^{\prime}, k^{2}\right)=(0.60,0.265)$, we see three local extremums in the quadrature result: a pair of local minima along the $y$-axis together with a saddle point at the origin $(0,0)$. These local extremums cannot be reproduced by the low-order analytic expansion such as $2 n=4$ or $2 n=8$; the local extremums show up only in the expansion of $2 n=12$ or higher, although the locations of the local minima along the $y$-axis are slightly different from the quadrature result. When $k^{2}$ becomes even smaller, the difference in the topology of the equipotential curves increases, as seen in Figure 2(e) where $\left(\alpha^{\prime}, k^{2}\right)=(0.60,0.260)$. A pair of local minima along the $y$-axis persists, but the origin $(0,0)$ becomes a local maximum and a pair of saddle points takes place along the $x$-axis. We confirmed that this topology is only reproduced when using the analytic expansion of disturbing function at our highest-order $(2 n=14)$, although the locations of the local extremums are slightly different from the quadrature result.

When $\alpha^{\prime}$ gets even larger and $k^{2}$ is in a certain range of moderate values, a pair of local minima show up along the $x$-axis inside the orbit-crossing lines together with a saddle point at the origin $(0,0)$. This is the case of Figure $2(\mathrm{f})$ when $\left(\alpha^{\prime}, k^{2}\right)=(0.80,0.304)$. The topology of the local extremums is reproduced by the analytic expansion of disturbing function at our highest-order $(2 n=14)$. However, we should note a point here. In the quadrature result of the rightmost panel in Figure 2(f), the pair of local minima along the $x$-axis appear when the value of $k^{2}$ is slightly different $\left(k^{2}=0.313\right)$ from the analytic result $\left(k^{2}=0.304\right)$. We have not yet figured out the cause of the discrepancy of the $k^{2}$ values that are required for the local minima to appear in the analytic expansion result and in the numerical quadrature result. It is possible that even higher-order analytic expansions of disturbing function $(2 n=16,18,20, \ldots)$ may diminish the discrepancy.

As a summary of this section, we say that the highorder analytic expansion of the doubly averaged disturbing function of CR3BP that has been presented in the present paper overall seems valid inside the orbit-crossing lines even when $\alpha$ (or $\alpha^{\prime}$ ) is large, as long as eccentricity of perturbed body is moderate. Under these conditions, local extremums of the doubly averaged disturbing function for CR3BP are quantitatively reproduced by the high-order analytic expansion.

\section{Comparison with Numerical Integration}

In Section 4 we inspected the validity of the high-order analytic expansion of the doubly averaged disturbing function by comparing its outcome with that from numerical quadrature. The numerical quadrature defined by the double integral (52) yields a numerical proxy of an integrable CR3BP system with one degree of freedom, and it is safe for us to directly compare the quadrature result with that from the analytic expansion of disturbing function. However, equations of motion of 
TABLE 1: Configuration of the numerical integration presented in Section 5. $\Delta t$ is nominal stepsize (days), $t_{\text {int }}$ is interval time of output (years), and $T$ is total integration period (million years). The parameter combinations of $\alpha$ (or $\alpha^{\prime}$ ) and $k^{2}$ from (a) to (f) are common to both in Figures 2, 3, 6, and 7.

\begin{tabular}{ccccccc}
\hline & $\alpha$ or $\alpha^{\prime}$ & $k^{2}$ & $m^{\prime} / m_{0}$ & $\Delta t$ & $t_{\text {int }}$ & $T$ \\
\hline (a) & $\alpha=0.90$ & 0.900 & $3.0404326 \times 10^{-6}$ & 2 & 500 & 10 \\
(b) & $\alpha=0.90$ & 0.400 & $3.0404326 \times 10^{-6}$ & 2 & 500 & 10 \\
(c) & $\alpha^{\prime}=0.70$ & 0.600 & $3.0404326 \times 10^{-6}$ & 2 & 500 & 10 \\
(d) & $\alpha^{\prime}=0.60$ & 0.265 & $4.7739597 \times 10^{-5}$ & 20 & 1000 & 200 \\
(e) & $\alpha^{\prime}=0.60$ & 0.260 & $4.7739597 \times 10^{-5}$ & 20 & 1000 & 200 \\
(f) & $\alpha^{\prime}=0.80$ & 0.313 & $9.5479194 \times 10^{-6}$ & 4 & 500 & 30 \\
\hline
\end{tabular}

three-body systems are generally not integrable, having shortterm oscillations and various mean motion resonances intact without being averaged out. To demonstrate the practical usefulness of our analytic expansion of the doubly averaged disturbing function, we carried out direct numerical integration of equations of motion using the same parameter combinations $\alpha$ (or $\alpha^{\prime}$ ) and $k^{2}$ as in the previous section. Based on the comparison, we will again give considerations on the validity of the high-order analytic expansion of the doubly averaged disturbing function for CR3BP.

5.1. Model and Method. We consider motion of small solar system bodies with infinitesimally small mass (asteroid, comet, etc.) undergoing gravitational perturbation from a major planet on a circular orbit. Using the same six sets of parameters in the framework of CR3BP as in Figure 2, we calculate orbit propagation of the small bodies by numerical integration for ten to two hundred million years. The parameter sets are tabulated in Table 1 . The main result of the numerical integration is presented and discussed in Section 5.2.

In Table 1 , mass ratios between perturbing body and central body $\left(m^{\prime} / m_{0}\right)$ for (a), (b), and (c) are close to the ratio of the Earth-Moon total mass and the solar mass $(\sim 1 / 314$ of the mass ratio between Jupiter and the Sun). The $\mathrm{m}^{\prime} / \mathrm{m}_{0}$ value for (f) is nearly $1 / 100$ of the mass ratio of Jupiter and the Sun. The reason for these small $\mathrm{m}^{\prime} / \mathrm{m}_{0}$ values is to avoid occurrence of orbital instability of the small body due to close encounters with the perturbing planet near the orbit-crossing lines. This is necessary because $\alpha$ and $\alpha^{\prime}$ are large in these four cases. We also chose small values for the stepsize of numerical integration $\Delta t$ for these cases by the same reason. As for (d) and (e), the $\mathrm{m}^{\prime} / \mathrm{m}_{0}$ value is close to $1 / 20$ of the mass ratio of Jupiter and the Sun. Since $\alpha^{\prime}$ is relatively small in these two cases, the probability of close encounters between perturbed and perturbing bodies is lower, and we can choose a larger mass ratio $m^{\prime} / m_{0}$ as well as larger stepsize $\Delta t$. Here, readers should recall the fact that the influence of the mass ratio $m^{\prime} / m_{0}$ is limited to the timescale of orbital evolution of perturbed body, and it does not affect the topology of relative orbit, particularly in the doubly averaged CR3BP. It is obvious from the function form of the doubly averaged disturbing function (19) or (24) where the perturber's mass serves just as a constant coefficient through $\mu^{\prime}$. Therefore, the trajectory shape of the perturbed body remains the same even if we change the mass of the perturber from $m^{\prime}$ to $p m^{\prime}$ (where $p$ is a positive real number); only the timescale of orbital evolution would change from $t$ to $t / p$. This is why we can arbitrarily change the mass ratio $m^{\prime} / m_{0}$ in numerical integration as long as what we need to know is the secular topology of trajectories of the perturbed body, not its time evolution sequence.

In the numerical integration, the semimajor axis of the perturbing body $a^{\prime}=5.2025217 \mathrm{AU}$ is common to all the cases, which is a substitute for Jupiter's semimajor axis. The perturber's mean anomaly (practically equivalent to mean longitude) $l^{\prime}$ is set to zero at time $t=0$. By the definition of CR3BP, the perturber's eccentricity $e^{\prime}$ and inclination $i^{\prime}$ are both set to zero. As for the perturbed body's initial orbital elements, semimajor axis $a$ is automatically determined from the value of $\alpha$ or $\alpha^{\prime}$. We set the initial values of eccentricity $e$ and argument of pericenter $g$ of perturbed body along the $x$ - and $y$-axis on the $(e \cos g, e \sin g)$ plane: we selected $e$ of perturbed body from $e=0$ to $e_{\max }$ with the interval of 0.01 . As for $g$, we used four values of $g=0, \pi / 2, \pi$, and $3 \pi / 2$. The values of the perturbed body's $i$ are automatically determined by relation (53). Initial values of the perturbed body's longitude of ascending node $(h)$ are set to 0 , and those for its initial mean anomaly are set to $\pi$.

The differential equation to be solved is the simple classical Newtonian equation of motion of the perturbed body. For the inner case, it is

$$
\frac{d^{2} \mathbf{r}}{d t^{2}}+\mu \frac{\mathbf{r}}{r^{3}}=\nabla R,
$$

with $\mu=\mathscr{G}_{m_{0}}$. For the outer case we just replace $R$ for $R^{\prime}$. As for the numerical integration scheme, we use the regularized mixed-variable symplectic method [44] based on the so-called Wisdom-Holman map [20,45]. In this scheme the stepsize of numerical integration is automatically reduced when a close encounter between two bodies happens, trying to keep the calculation accuracy high. The $\Delta t$ values listed in Table 1 are the nominal stepsizes used when no close encounter takes place between bodies. We have implemented this scheme based on the code SWIFT [44] and used it in our previous studies where close encounters between asteroids and terrestrial planets take place very often [46, 47], as well as where almost no close encounter happens [48, 49]. We have confirmed that our implementation of this scheme does not have a practical problem in accuracy or efficiency when applied to these dynamical systems. We adopt the Gaussian units where the Gaussian gravitational constant $\sqrt{\mathscr{G}}=0.01720209895$, the unit of time is an ephemeris day, and the unit of length is the astronomical unit (AU), so that the mass of the primary body becomes unity (e.g., $[16,50])$.

5.2. Integration Result. We show the main results of our numerical integration in Figure 3 whose parameters are described in Table 1 . The perturbed body's $(e \cos g, e \sin g$ ) values are plotted on the panels by black and green dots (see below for the reason for this distinction) with the fixed output interval $t_{\text {int }}$ designated in Table 1 . We chose $t_{\text {int }}$ empirically so that the output data amount does not get too 


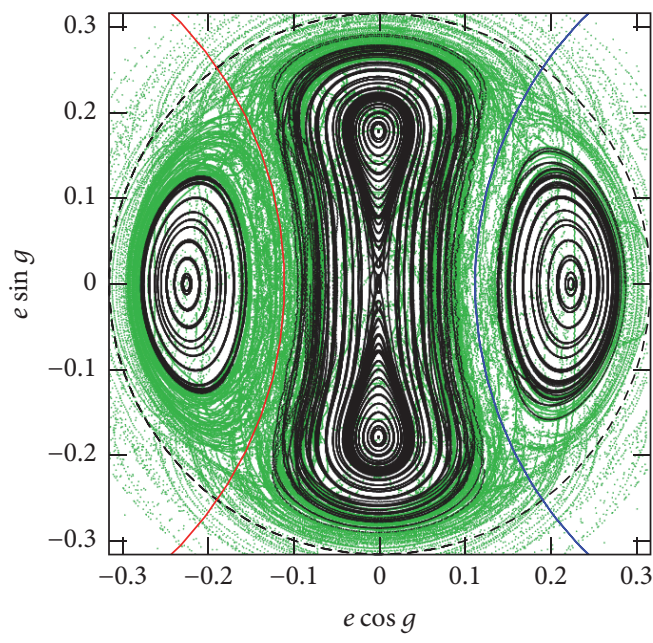

(a)

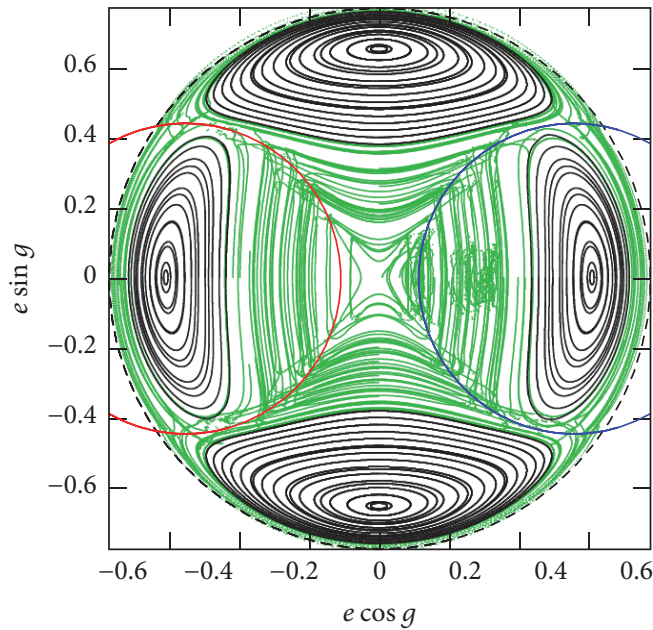

(b)

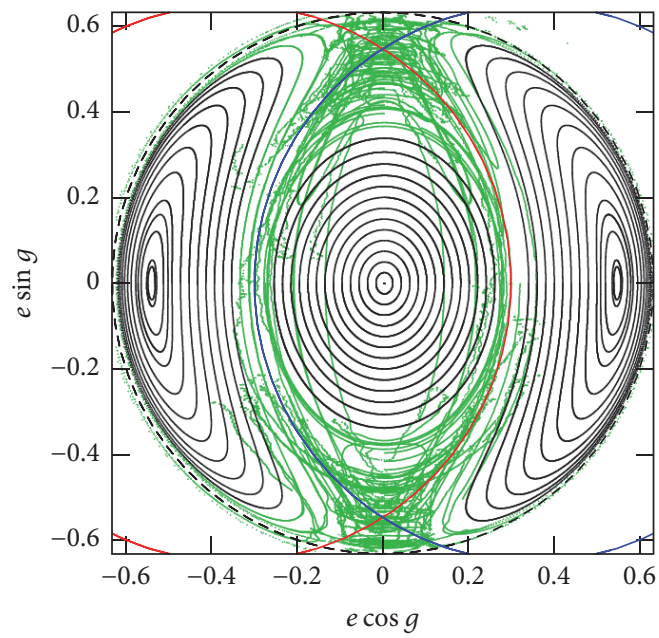

(c)

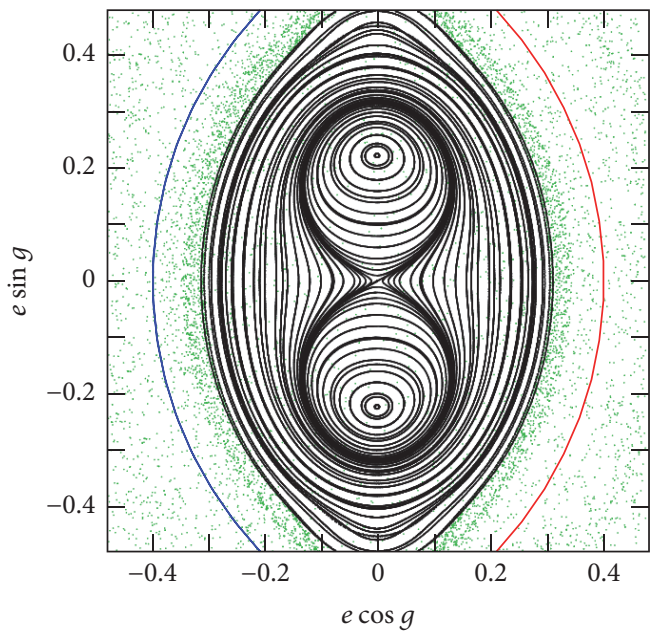

(d)

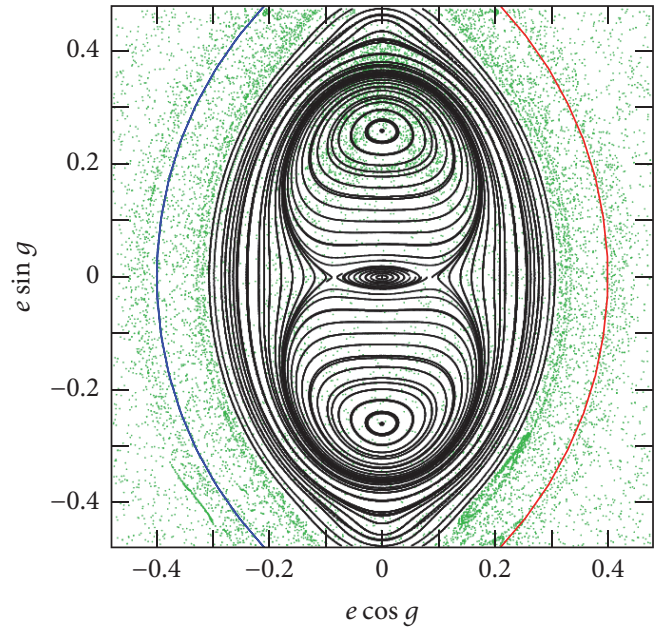

(e)

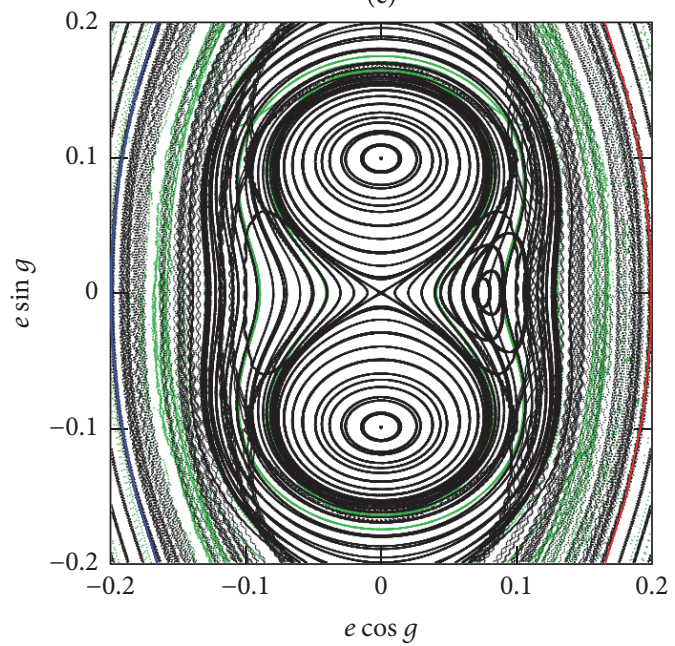

(f)

FIGURE 3: Plots of the perturbed body's $(e \cos g, e \sin g$ ) values obtained from the direct numerical integration whose parameters are listed in Table 1. As for the meaning of the black dashed circles and of the red and blue partial circles, see the caption of Figure 2 . The green dots represent the trajectories of bodies that experienced orbital instability during the integration period. Stable trajectories are drawn by black dots. 
large, while avoiding any kind of aliasing in the plots. The following settings are all common to both Figures 2 and 3: the coordinate system $(x, y)=(e \cos g, e \sin g)$, the ranges of $x$ - and $y$-axis, meaning of the black dashed circles, and that of the red and blue partial circles.

When inspecting Figure 3, we would like readers to notice two points: One is that we drew trajectories of perturbed bodies in black only when their orbital motion remains stable from the beginning to the end of the integration period $T$. This is to facilitate the comparison between the quadrature result and the integration result. Here we define the "stable" orbital motion if the semimajor axis a does not exhibit any major changes throughout the integration period $T$. Otherwise we categorize the orbital motion as "unstable." This categorization is done by the naked eye, plotting the time sequence of $a$ for all the perturbed bodies used in the integration ( 320 bodies per panel, 1920 bodies in total). Browsing through all the plots of $a$, we subjectively distinguish "stable" and "unstable" orbits. Hence there can be some ambiguity in the categorization. However, let us say that the difference between stable and unstable orbits is rather clear in Figure 3, and the subjective distinction between the black and the green trajectories does not affect the discussion here.

The second point is that we did not draw some of the trajectories that are largely overlapped with others. This is to prevent the panels from becoming too busy with too many similar trajectories, as well as to keep the figure file size reasonably small. As we mentioned before, starting points of the numerical integration are aligned on the $x$ - and $y$-axis as $g=0, \pi / 2, \pi, 3 \pi / 2$ with a fixed interval of eccentricity. Due to the intrinsic symmetry that the disturbing function has, different initial condition can generate almost identical trajectories. For example, in Figure 3(c) the initial conditions $(e, g)=(0.03,0)$ and $(e, g)=(0.03, \pi)$ generate almost identical trajectories. For eliminating redundancy in figure and reducing the file size, we did not draw the trajectory from the initial condition $(e, g)=(0.03, \pi)$ and just drew that from $(e, g)=(0.03,0)$. We carried out this kind of selection of overlapped trajectories with great care so that our subjective selection never alters general topological patterns in the panels.

Comparing Figures 3 and 2, we say that their agreement is overall good. In Figure 3(a) for the inner CR3BP, existence of stationary points at $g= \pm \pi / 2$ is confirmed by the numerical integration. The eccentricity value of the stationary points is close to what the analytic expansion and the numerical quadrature had yielded in Figure 2(a). Existence of stationary points at $g=0, \pi$ outside the orbit-crossing lines is also confirmed, although most of the trajectories near the orbitcrossing lines become unstable due to close encounters with the perturbing body.

Similarly in Figure 3(b), existence of stationary points at $g= \pm \pi / 2$ as well as those at $g=0, \pi$ is confirmed by the numerical integration. There must be a saddle point at the origin $(x, y)=(0,0)$, but most of the trajectories near the origin are unstable in Figure $3(\mathrm{~b})$ due to the proximity to the orbit-crossing lines, and it is not easy to visually confirm it.
In Figure 3(c) for the outer CR3BP, existence of a stationary point at the origin $(x, y)=(0,0)$ as well as those at $g=0, \pi$ is confirmed by the numerical integration. It is clearly seen that trajectories can easily become unstable around the two intersections of the red and blue orbit-crossing lines along the $y$-axis.

In Figures 3(d) and 3(e), the existence of stationary points is again confirmed by the numerical integration. We see a pair of stationary points at $g= \pm \pi / 2$ in both panels. The origin $(x, y)=(0,0)$ in Figure $3(\mathrm{~d})$ makes a saddle point, as suggested by the quadrature (Figure $2(\mathrm{~d})$ ). The origin $(0,0)$ in Figure 3(e) makes a local maximum, which is also consistent with the quadrature result (Figure 2(e)). A pair of saddle points seen along the $x$-axis in Figure $3(\mathrm{e})$ has also been suggested by the quadrature (Figure 2(e)). Although the location of the saddle points seen in Figure 3(e) is slightly closer to the origin $(0,0)$ compared with that in Figure 2(e), we would say they are consistent. In both the panels of Figures $3(\mathrm{~d})$ and 3(e), trajectories get unstable and the green dots are scattered as they approach the orbit-crossing lines.

As we have seen in Figures 3(a)-3(e), the result from the direct numerical integration of the equations of motion overall agrees well with the result from the numerical quadrature, as well as with that from the analytic expansion of the doubly averaged disturbing function. We thus say that this comparison largely justifies the validity of use of the analytic expansion of the doubly averaged disturbing function for CR3BP presented in Section 3.

However, in Figure 3(f) we encounter with a typical system where the use of doubly averaged disturbing function, either through analytic expansion or quadrature, is not appropriate. As is clearly seen, the topological pattern of trajectories in Figure 3(f) is very different from the equipotential contours shown in Figure 2(f). A pair of stationary points shows up at $g= \pm \pi / 2$ in Figure 3(f) that we do not see in Figure 2(f). Also, although we see stationary points along the $x$-axis in Figure 3(f), the shape of the trajectories is fairly different from what is observed in Figure 2(f). We then plotted the whole phase space for this system $\left(e \leq e_{\max }\right)$ in Figure 4. As we see, the trajectories outside the orbit-crossing lines are very similar between Figure 4(a) (quadrature) and Figure 4(b) (integration). But the complicated trajectory pattern inside the orbit-crossing lines takes place only in the numerical integration result (Figure 4(b)).

So far we have not completely figured out the cause of the difference in trajectory patterns between Figures 2(f) and $3(\mathrm{f})$. We have confirmed that there is no problem in accuracy of the numerical integration that is produced in Figure 3. As far as we have been able to investigate, the apparently peculiar trajectories seen in Figure 3(f) are possibly related to the existence of a mean motion resonance. We selected some of the periodic orbits observed inside the orbit-crossing lines in Figure 4(b) and plotted them in separate panels in Figure 5 (the panels in its left column). For each of the periodic orbits, we calculated an argument $5 \lambda^{\prime}-7 \lambda+2 g$, where $\lambda^{\prime}$ is mean longitude of the perturbing body and $\lambda$ is that of the perturbed body. As a result, we found that in the top two trajectories (Figures 5(a) and 5(b)) the argument $5 \lambda^{\prime}-7 \lambda+2 g$ librates around $\pi$, although the libration 


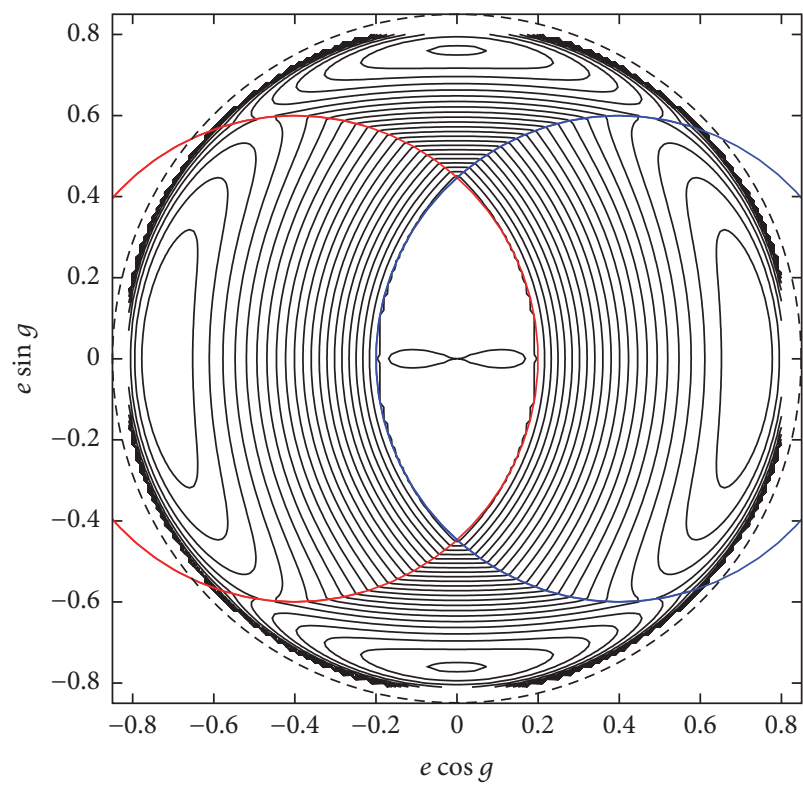

(a)

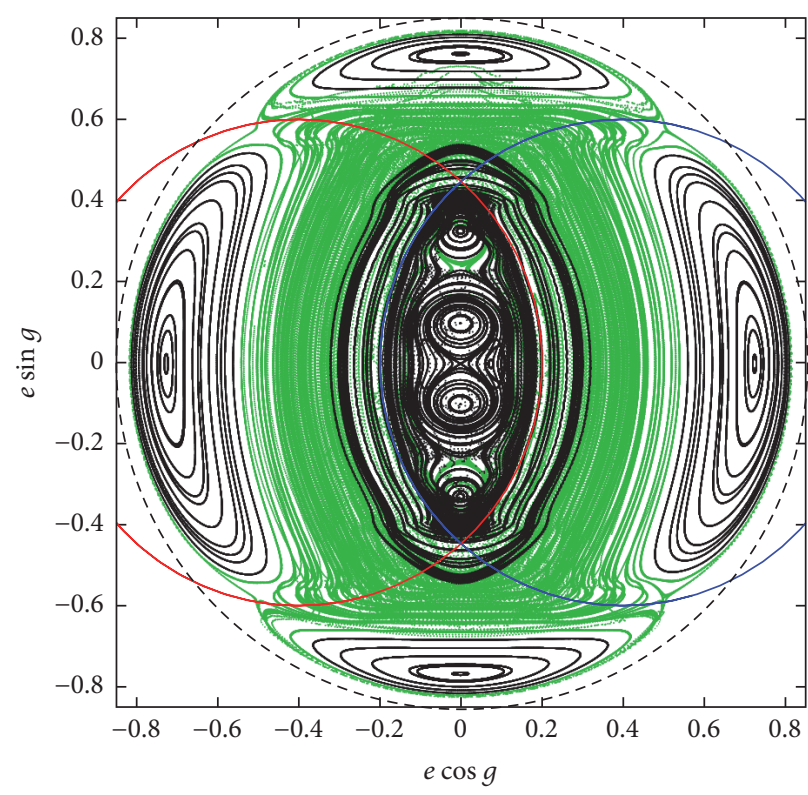

(b)

Figure 4: (a) Extended plot of Figure 2(f) up to $e=e_{\max }$ (numerical quadrature). (b) Extended plot of Figure 3(f) (numerical integration). Note that we set the contour interval in (a) ten times larger than in Figure 2(f) for a better visibility of plots. The interval of initial eccentricity in (b) is twice larger than in Figure 3(f) for the same reason.

amplitude is relatively large (see the panels in the middle column of Figure 5). This indicates that the systems in Figures 5(a) and 5(b) are trapped in the 5:7 mean motion resonance. System (c) seems close to this resonance too. It is well known that the existence of strong mean motion resonance can substantially change the shape of a disturbing potential, together with the location of local extremums, compared with nonresonant cases (e.g., [51-53]). To deal with resonant systems like Figure 3(f) rigorously, we would need to employ different methods that are particular to each of the resonant systems (e.g., [54, 55]).

Although all the five perturbed bodies whose trajectories are shown in Figure 5 started with the same initial semimajor axis $\left(a_{0}=6.503152 \mathrm{AU}\right)$, we see a systematic difference in the average value of the semimajor axis $a$ between $5(\mathrm{a}-\mathrm{c})$ and $5(\mathrm{~d}$, e) (see the panels in the right column of Figure 5). The shortterm oscillation of $a$ with relatively large amplitude seen in Figures 5(a),5(b), and 5(c) is probably related to the $5: 7$ mean motion resonance. It is possible that the oscillation of $a$ seen in Figures 5(d) and 5(e) is also related to this resonance or others that we have not yet found.

We will pursue the above problem in our forthcoming publications. Several possibilities might account for the peculiar trajectories seen in Figure 4(b), such as the effect of high-order mean motion resonance (e.g., [56]) and its combination with the Lidov-Kozai libration (e.g., [57]). A kind of secondary resonance may be embedded in this system (e.g., [58]). We should be aware that more and more mean motion resonances show up as $\alpha$ or $\alpha^{\prime}$ approaches 1 (e.g., [59]), and treatment of such systems would need greater care.

For readers' interest, we also plotted trajectories $(\sin i \cos h, \sin i \cos h)$ of the perturbed body where $i$ is its inclination and $h$ is its longitude of ascending node. The six panels from (a) to (f) in Figure 6 correspond to each in Figure 3 with the same color pattern (stable orbits are drawn in black, and unstable orbits are drawn in green). Recalling the fact that the theoretical smallest value of $\cos i$ takes place when $e=0$ as $|\cos i|_{\min }=k$ (see Section 4), it is clear that the theoretical largest value of $\sin i$ is $|\sin i|_{\max }=\sqrt{1-k^{2}}$ when $e=0$ (note that this value happens to be the same as $\left.e_{\max }\right)$. We drew the outer boundary $|\sin i|_{\max }=\sqrt{1-k^{2}}$ by the black dashed circles in Figure 6.

In the doubly averaged CR3BP system that we now consider, eccentricity $e$ and inclination $i$ are correlated with each other through the definition of $k^{2}$ in (53). However, since longitude of ascending node $h$ is not included in the disturbing function $R, h$ does not have a particular correlation with eccentricity $e$, inclination $i$, or argument of pericenter g. The panels of Figures 6(b) and 6(c) typically exemplify this circumstance where we see the trajectories of perturbed bodies which are not closed on the $(\sin i \cos h, \sin i \cos h)$ plane; $i$ and $h$ are not correlated, and these trajectories do not compose equipotential contours.

For more demonstrations of the time variation of $i$ and $h$, we picked one body per each of the panels from Figure 6(a) to Figure 6(f) and showed the time variation of their $e, i, g$, and $h$ in Figure 7. Initial values of $(e, g)$ of each of the bodies are written in the caption of Figure 7. Our choice of the particular body among the 320 bodies in each of the panels is almost arbitrary: we basically chose the bodies whose argument of pericenter librates around $g=\pi / 2$ or $0(a, b, c, d$, and $f)$, but we chose a body whose argument of pericenter circulates from 0 to $2 \pi$ (e). 

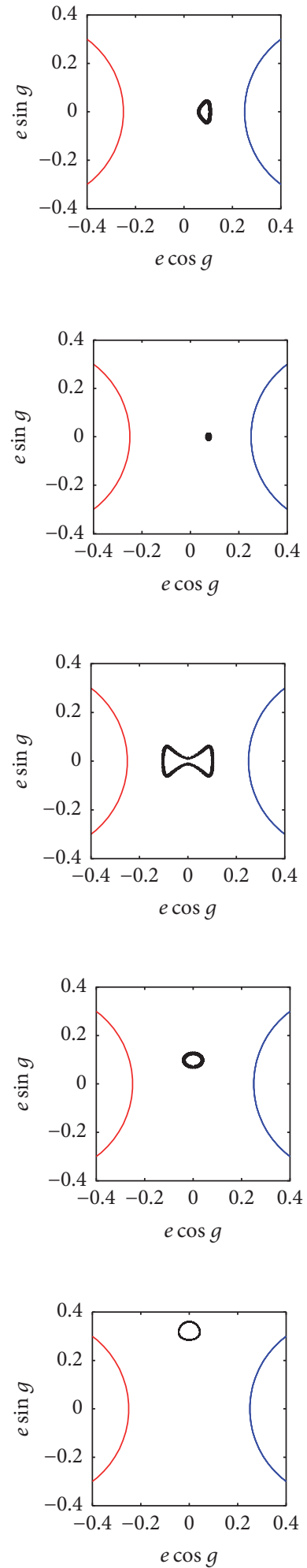

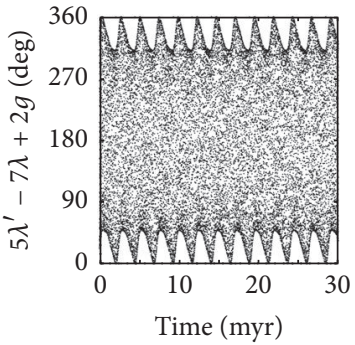

(a)

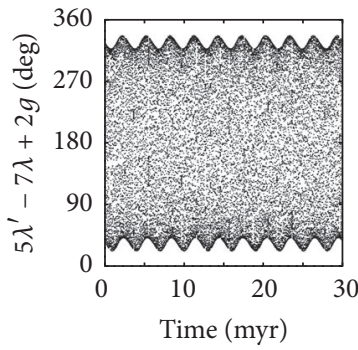

(b)

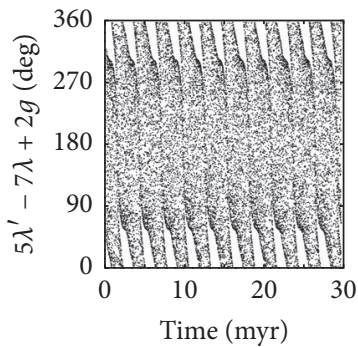

(c)
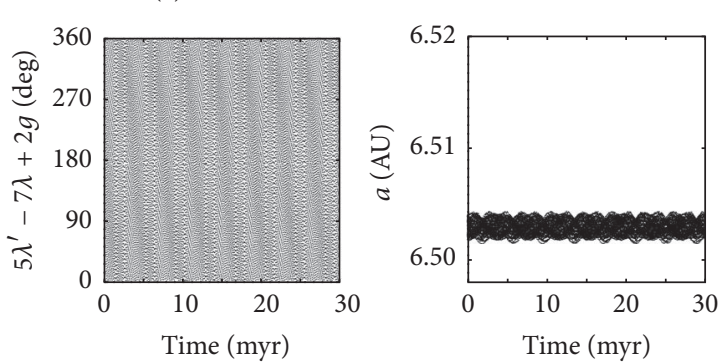

(d)
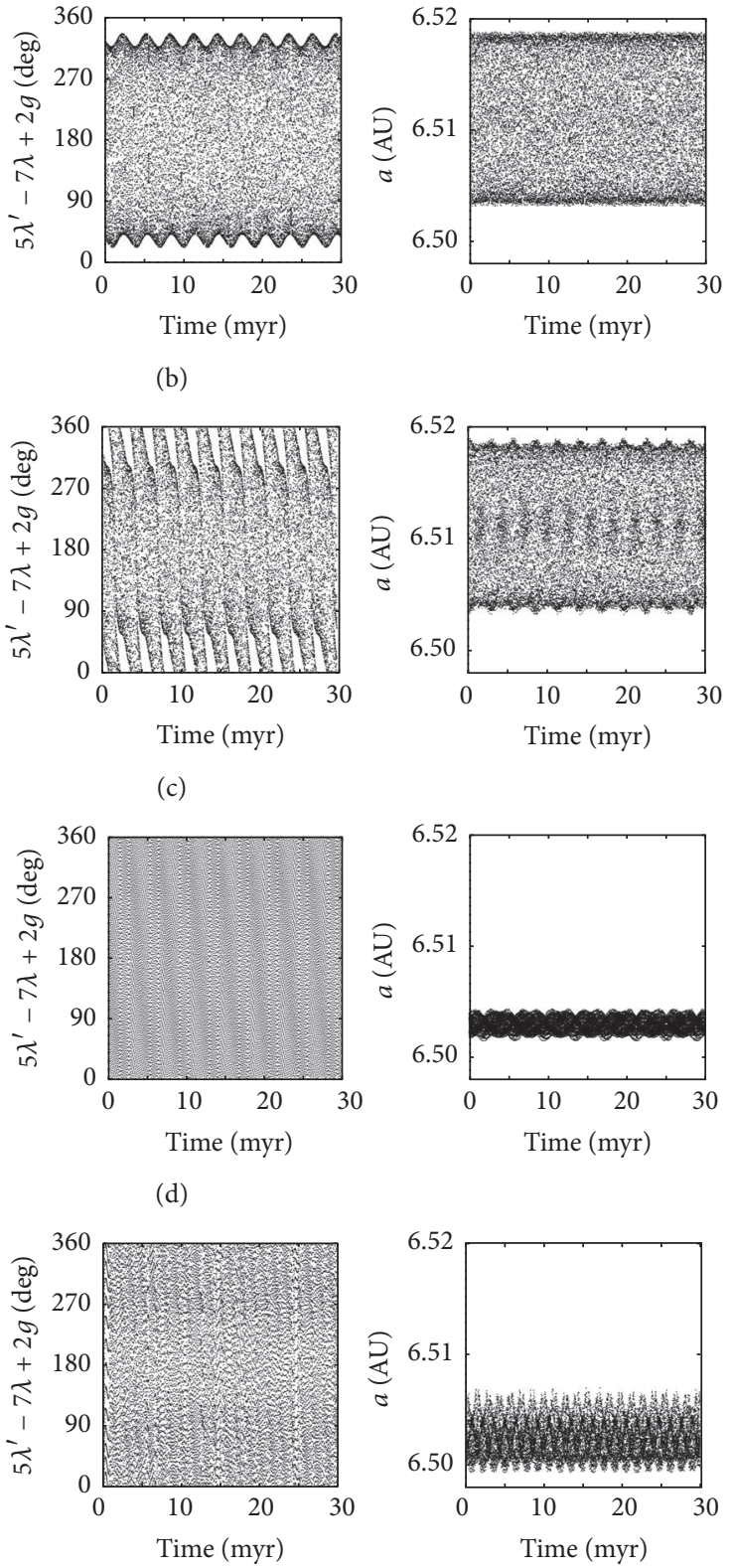

(e) 


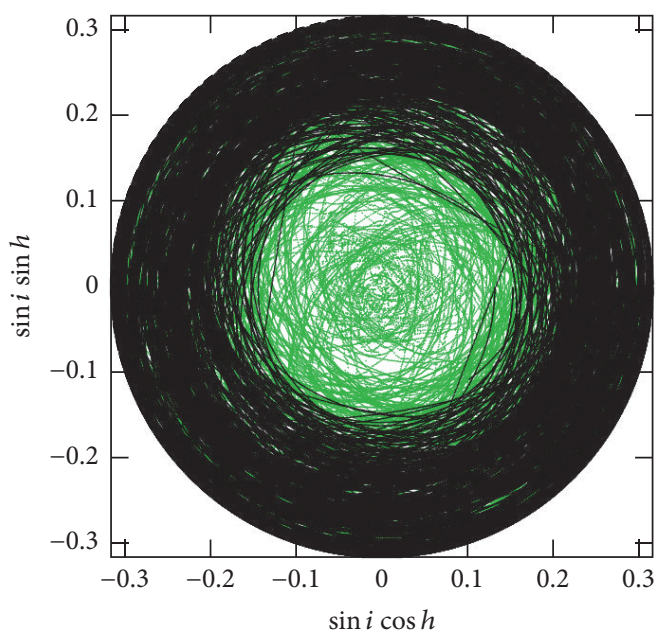

(a)

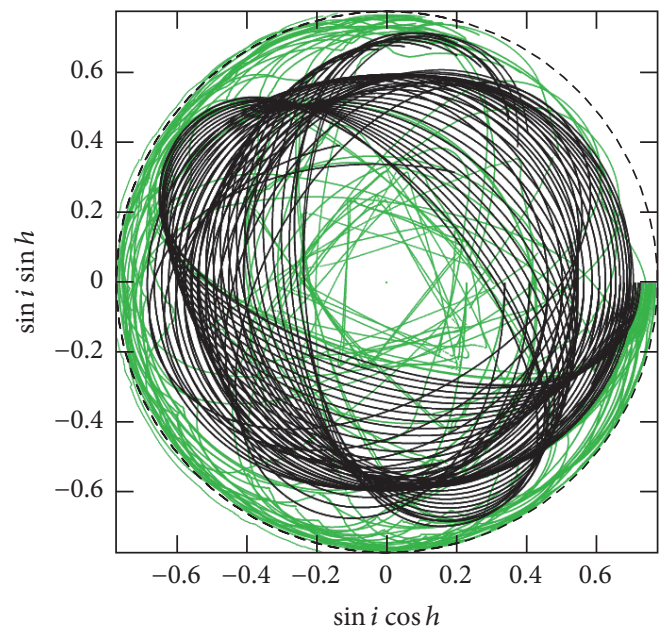

(b)

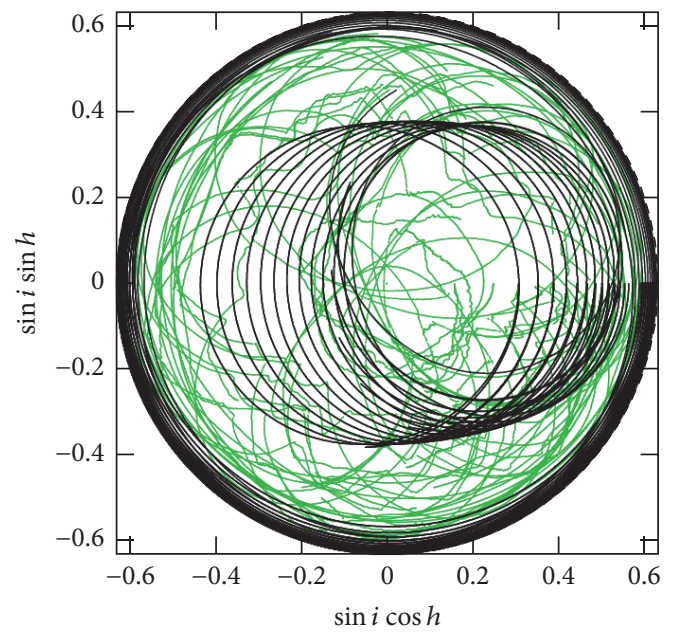

(c)

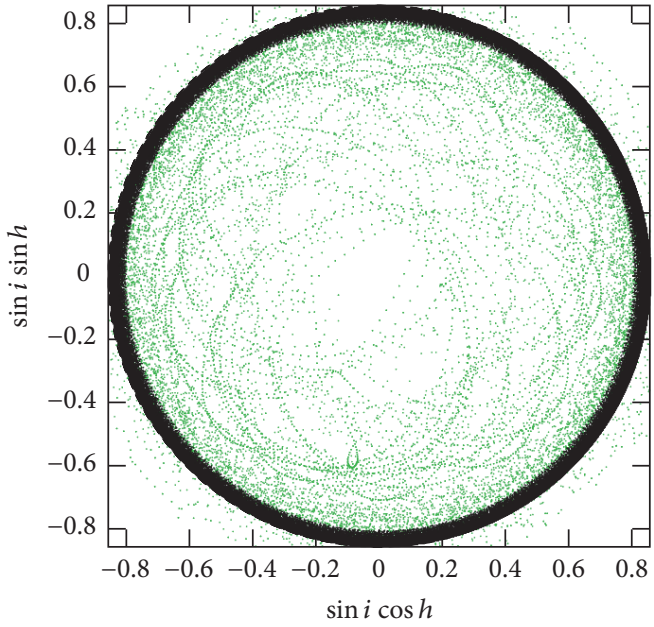

(d)

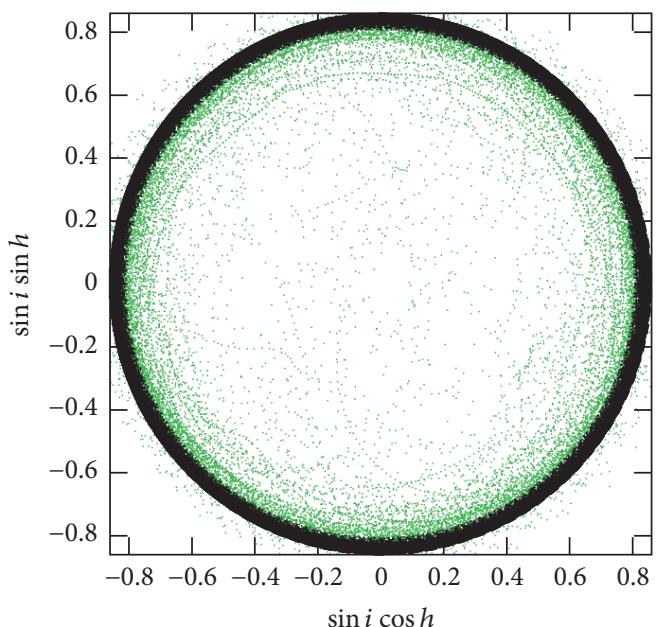

(e)

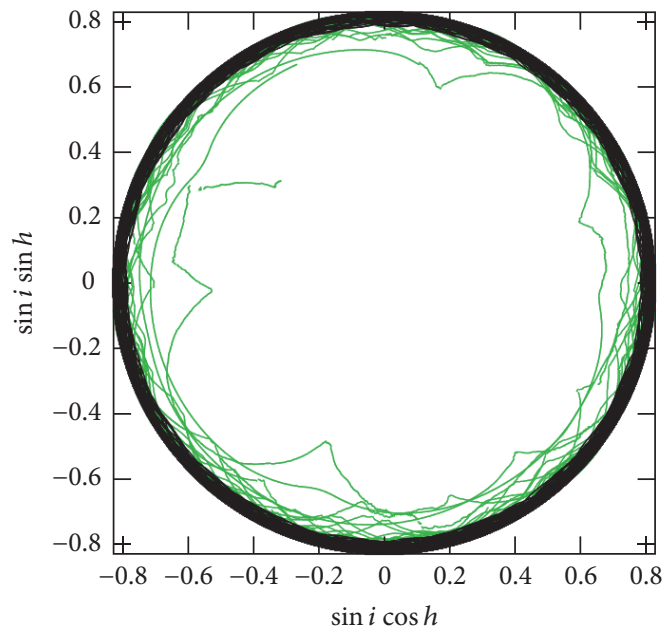

(f)

Figure 6: Plots of the perturbed body's $(\sin i \cos h, \sin i \sin h)$ values obtained from the direct numerical integration whose parameters are listed in Table 1 . The black dashed circles represent the theoretical maximum $\sin i$ value (which is the same as $e_{\max }$ ). Meanings of the black dots and the green dots remain the same as in Figure 3. 

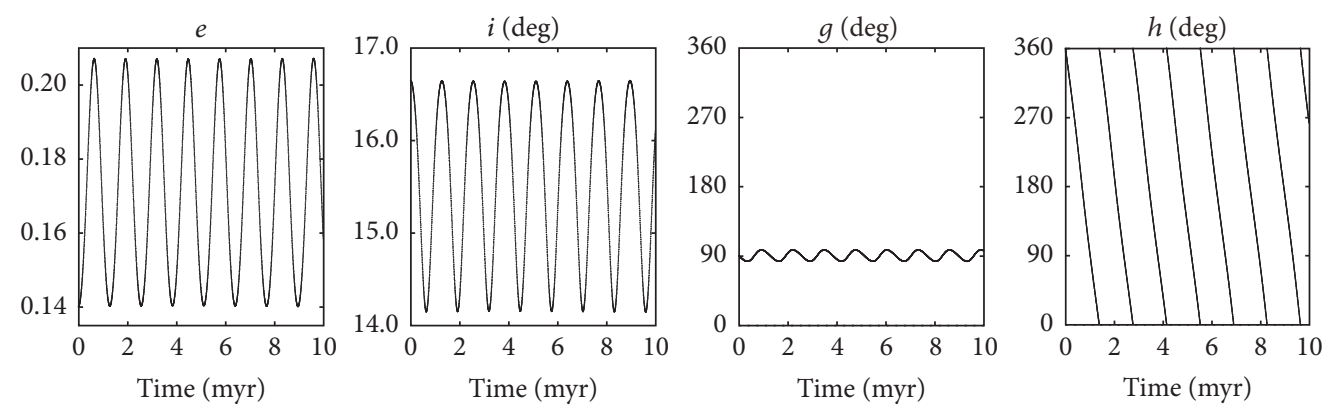

(a)
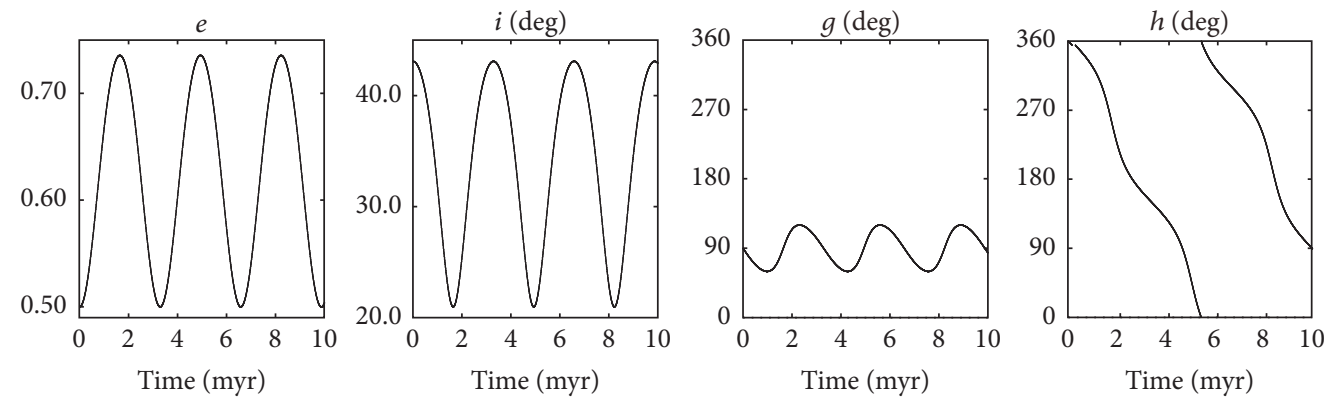

(b)
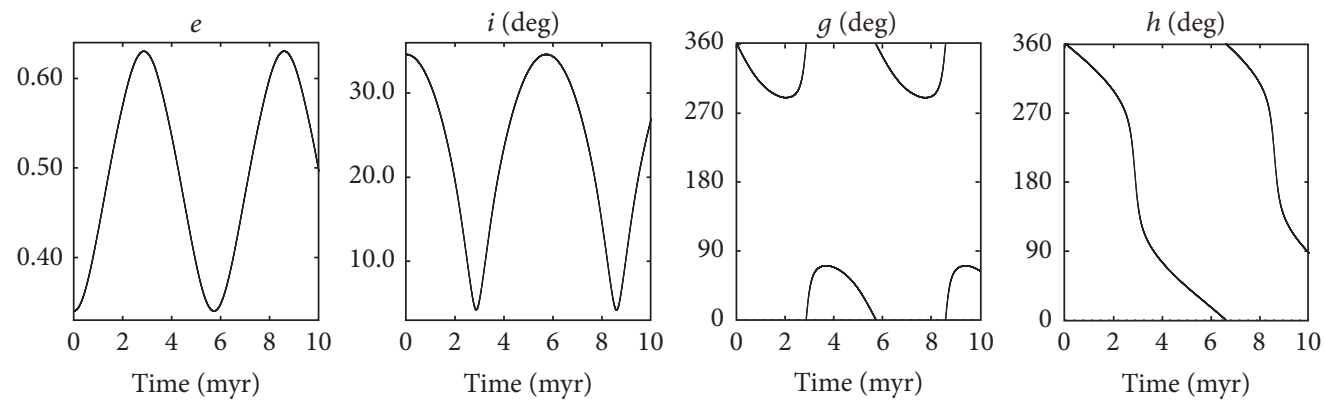

(c)
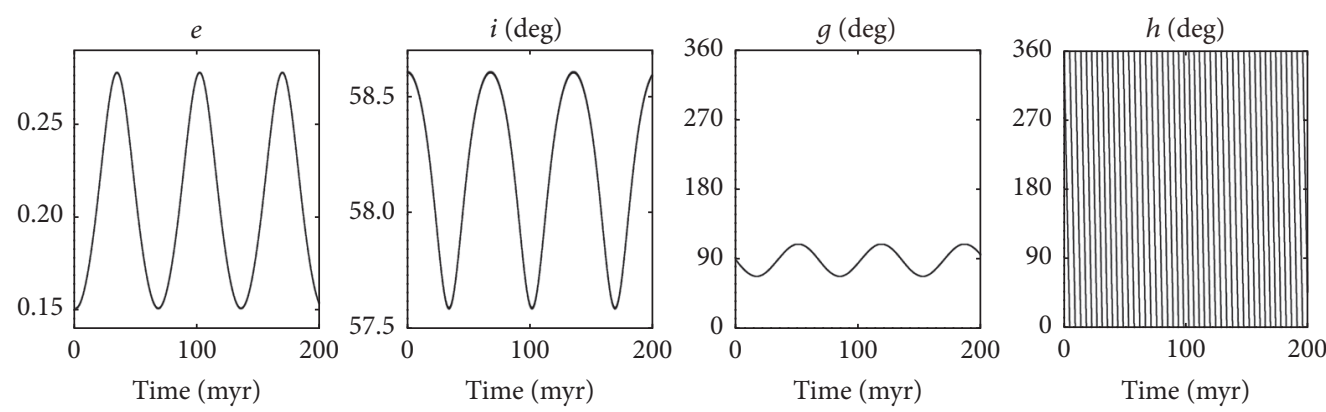

(d)
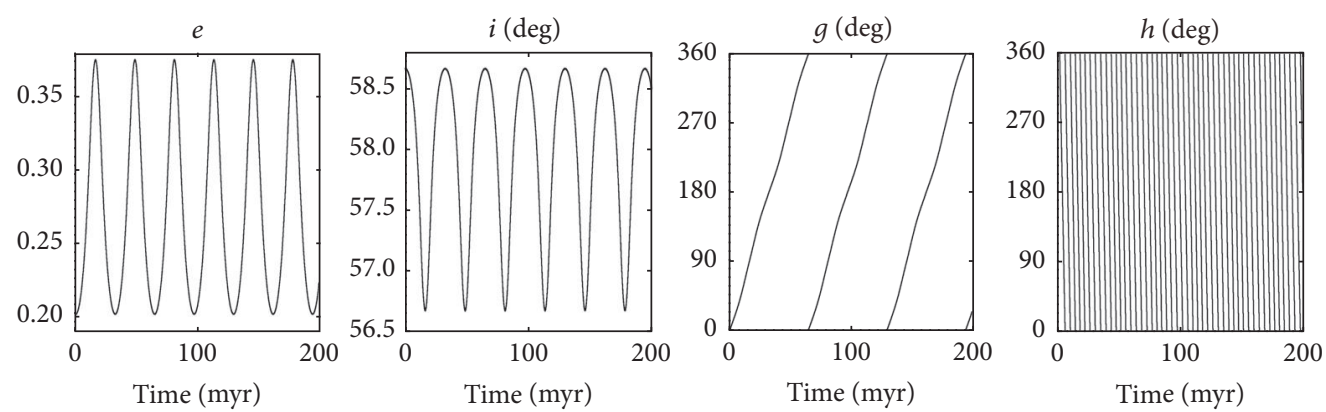

(e)

Figure 7: Continued. 


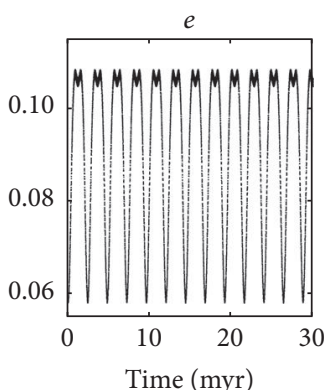

Time (myr)

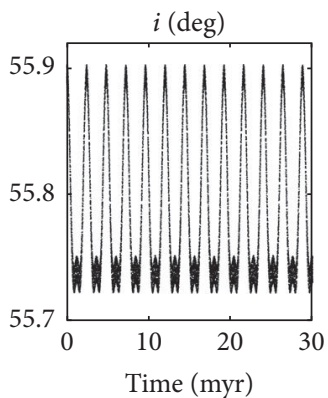

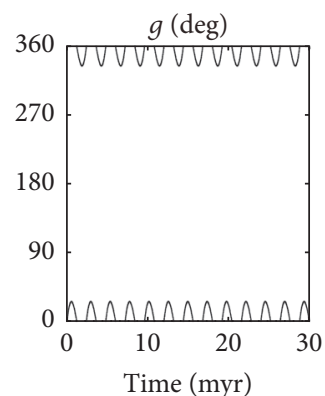

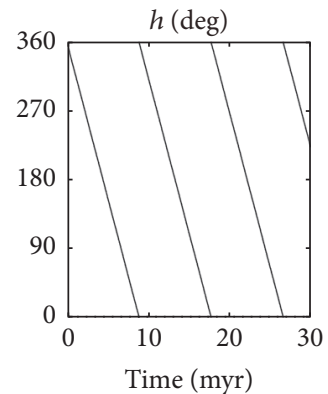

(f)

Figure 7: Variation of $e, i, g$, and $h$ of representative bodies in Figures 6(a)-6(f). Initial values of $e$ and $g$ for each body are as follows: (a) $(e, g)=(0.14, \pi / 2),(\mathrm{b})(e, g)=(0.50, \pi / 2),(\mathrm{c})(e, g)=(0.34,0),(\mathrm{d})(e, g)=(0.15, \pi / 2),(\mathrm{e})(e, g)=(0.20,0)$, and $(\mathrm{f})(e, g)=(0.06,0)$. Note that the unit of $g$ and $h$ is degree, not radian, for consistency with the unit of $i$. All the elements are plotted with a time interval of 1500 years and are calculated on the heliocentric coordinate centered on the central mass.

The panels in Figure 7 immediately tell us the correlation between eccentricity $e$ and inclination $i$, which is an obvious outcome of the preservation of $k^{2}$ in (53). The argument of pericenter $g$ is correlated to them, as we have seen in the study of the doubly averaged disturbing function in Section 3. The rightmost panels show the fact that only longitude of ascending node $h$ has no correlation with other elements; it shows a monotonic decrease in all the panels.

We do not show the analytic counterpart of Figure 6 or Figure 7 because they are out of the scope of the present paper. Readers with particular interest may consult classic literature such as Moiseev [34, 60] or Lidov [2] for how to formally obtain analytic solutions of time evolution of $h$. For example, in Lidov [2], the author uses the mutual dependence between $e, i$, and $g$ and constructs a definite integral for quadrature that formally depicts the function form of $h$ depending on time. Incidentally, if we limit ourselves to the doubly averaged inner CR3BP at the quadruple-order analytic approximation $(2 n=2$; see Section 3.2), analytic solution of $h$ and that of $g$ (hence those of $e$ and $i$ ) using elliptic functions have already been derived in much of the literature (e.g., $[3,5-7,61,62]$ ).

\section{Summary and Discussion}

Through the comparison of the results obtained from analytic expansion of the doubly averaged disturbing function, numerical quadrature, and direct numerical integration of the equations of motion, overall we validated the use of our high-order analytic expansion of the doubly averaged disturbing function both for the inner and outer CR3BP, even when the semimajor axis ratio $\alpha$ (or $\alpha^{\prime}$ ) is large. Our result tells us that the high-order analytic expansion is not applicable in several circumstances: For example, when a perturbed body's eccentricity approaches its theoretical largest value, when orbits of perturbed and perturbing bodies cross, and when strong mean motion resonance is at work. Particularly, occurrence of strong mean motion resonance can significantly change global topology of disturbing potential, and the doubly averaged method itself goes out of use. Nevertheless, we think our original objective to analytically exemplify dynamical characteristics of doubly averaged disturbing function for CR3BP in this paper is largely fulfilled. We thus conclude that the high-order analytic expansion of the doubly averaged disturbing function is suitable for expository purposes to account for the dynamical characteristics of secular CR3BP even when $\alpha$ (or $\alpha^{\prime}$ ) is large, as long as the system is free from serious mean motion resonance.

Let us just mention a point regarding one limitation of the high-order analytic expansion of the doubly averaged disturbing function. As seen in Sections 4 and 5, sometimes the locations of local extremums of the analytically expanded doubly averaged disturbing function differ from those obtained by the numerical quadrature and numerical integration, particularly in the outer problem such as in Figure 2(d) or 2(e). In the outer case, the factor $\left(1-e^{2}\right)^{-2 n+1 / 2}$ in (24) substantially enhances the magnitude of high-order terms in the analytically expanded disturbing function when eccentricity $e$ is large. For example, when $e=0.5$ the factor becomes $\sim 8.65(2 n=8), \sim 27.3(2 n=12)$, and $\sim 48.6(2 n=$ $14)$. When $e=0.8$, the factor reaches $\sim 2127(2 n=8), \sim 126626$ $(2 n=12)$, and $\sim 977049(2 n=14)$, possibly causing artificial oscillation of the higher-order analytic terms in the large-e region.

Incidentally, we would like readers to be aware of a recent study that revealed that solutions of the three-body problem obtained from the double averaging procedure can substantially deviate from true solution due to accumulation of the effect of short-term oscillation [63]. This can happen particularly when the mass of the perturbing body is not negligibly small compared to the primary mass.

A Maple script that yields our analytic expansion in the present paper (see (25)-(51)) is available from the author upon request.

\section{Competing Interests}

The author declares no competing financial interests associated with this article.

\section{Acknowledgments}

The author has benefited from stimulating enlightenment through discussions with Katsuhito Ohtsuka, Arika Higuchi, 
Akihiko Fujii, Ruslan Salyamov, Fumi Yoshida, and Renu Malhotra. Detailed and constructive review by Yolande McLean has considerably improved the English presentation of this paper. Algebraic manipulations with Maple, numerical quadrature operations, and orbit propagation by numerical integration carried out in the present paper were all performed at Center for Computational Astrophysics (CfCA), National Astronomical Observatory of Japan (NAOJ). This study is supported by a pair of JSPS Kakenhi Grants, JP25400458/2013-2016 and JP16K05546/2016-2018.

\section{References}

[1] V. Szebehely, Theory of Orbits, Academic Press, New York, NY, USA, 1967.

[2] M. L. Lidov, "The evolution of orbits of artificial satellites of planets under the action of gravitational perturbations of external bodies," Planetary and Space Science, vol. 9, no. 10, pp. 719-759, 1962.

[3] Y. Kozai, "Secular perturbations of asteroids with high inclination and eccentricity," The Astronomical Journal, vol. 67, pp. 591$598,1962$.

[4] I. Shevchenko, The Lidov-Kozai Effect: Applications in Exoplanet Research and Dynamical Astronomy, vol. 441 of Astrophysics and Space Science Library, Springer, Dordrecht, The Netherlands, 2017.

[5] H. Kinoshita and H. Nakai, "Secular perturbations of fictitious satellites of Uranus," Celestial Mechanics \& Dynamical Astronomy, vol. 52, no. 3, pp. 293-303, 1991.

[6] H. Kinoshita and H. Nakai, "Analytical solution of the Kozai resonance and its application," Celestial Mechanics \& Dynamical Astronomy, vol. 75, no. 2, pp. 125-147, 1999.

[7] H. Kinoshita and H. Nakai, "General solution of the Kozai mechanism," Celestial Mechanics \& Dynamical Astronomy, vol. 98, no. 1, pp. 67-74, 2007.

[8] V. I. Prokhorenko, "A geometric study of solutions to restricted circular double-averaged three-body problem," Cosmic Research, vol. 39, no. 6, pp. 583-593, 2001.

[9] M. Nagasawa, S. Ida, and T. Bessho, "Formation of hot planets by a combination of planet scattering, tidal circularization, and the Kozai mechanism," The Astrophysical Journal, vol. 678, no. 1, pp. 498-508, 2008.

[10] E. B. Ford, B. Kozinsky, and F. A. Rasio, "Secular evolution of hierarchical triple star systems," The Astrophysical Journal, vol. 535, no. 1, pp. 385-401, 2000.

[11] B. Katz, S. Dong, and R. Malhotra, "Long-term cycling of KozaiLidov cycles: extreme eccentricities and inclinations excited by a distant eccentric perturber," Physical Review Letters, vol. 107, no. 18, Article ID 181101, 2011.

[12] S. Naoz, W. M. Farr, Y. Lithwick, F. A. Rasio, and J. Teyssandier, "Secular dynamics in hierarchical three-body systems," Monthly Notices of the Royal Astronomical Society, vol. 431, no. 3, pp. 2155-2171, 2013.

[13] S. Naoz, "The eccentric Kozai-Lidov effect and its applications," Annual Review of Astronomy and Astrophysics, vol. 54, no. 1, pp. 441-489, 2016.

[14] J. Laskar and G. Boué, "Explicit expansion of the three-body disturbing function for arbitrary eccentricities and inclinations," Astronomy \& Astrophysics, vol. 522, no. 5, article A60, 2010.

[15] C. D. Murray and S. F. Dermott, Solar System Dynamics, Cambridge University Press, Cambridge, UK, 1999.
[16] D. Brouwer and G. M. Clemence, Methods of Celestial Mechanics, Academic Press, New York, NY, USA, 1961.

[17] J. M. Danby, Fundamentals of Celestial Mechanics, WillmannBell, Richmond, Va, USA, 2nd edition, 1992.

[18] H. C. Plummer, An Introductory Treatise on Dynamical Astronomy, Dover, New York, NY, USA, 1960.

[19] W. M. Smart, Celestial Mechanics, Longmans, London, UK, 1954.

[20] J. Wisdom and M. Holman, "Symplectic maps for the $N$-body problem," The Astronomical Journal, vol. 102, no. 4, pp. 1528$1538,1991$.

[21] P. Saha and S. Tremaine, "Long-term planetary integration with individual time steps," The Astronomical Journal, vol. 108, no. 5, pp. 1962-1969, 1994.

[22] R. S. Harrington, "Dynamical evolution of triple stars," The Astronomical Journal, vol. 73, pp. 190-194, 1968.

[23] Y. Krymolowski and T. Mazeh, "Studies of multiple stellar systems-II. Second-order averaged Hamiltonian to follow long-term orbital modulations of hierarchical triple systems," Monthly Notices of the Royal Astronomical Society, vol. 304, no. 4, pp. 720-732, 1999.

[24] H. Beust and A. Dutrey, "Dynamics of the young multiple system GG Tauri. II. Relation between the stellar system and the circumbinary disk," Astronomy \& Astrophysics, vol. 446, pp. 137-154, 2006.

[25] J. P. S. Carvalho, R. V. de Moraes, A. F. B. A. Prado, and O. C. Winter, "Analysis of the secular problem for triple star systems," Journal of Physics: Conference Series, vol. 465, no. 1, Article ID 012010, 2013.

[26] R. A. Broucke, "Expansion of the third-body disturbing function," Journal of Guidance, Control, and Dynamics, vol. 4, no. 3, pp. 346-348, 1981.

[27] R. A. Mardling, "New developments for modern celestial mechanics-I. General coplanar three-body systems. Application to exoplanets," Monthly Notices of the Royal Astronomical Society, vol. 435, no. 3, pp. 2187-2226, 2013.

[28] M. E. Bailey, "Is there a dense primordial cloud of comets just beyond Pluto?" in Proceedings of the Asteroids, Comets, Meteors, pp. 383-386, Uppsala Universitet, Uppsala, Sweden, June 1983.

[29] T. Quinn, S. Tremaine, and M. Duncan, "Planetary perturbations and the origin of short-period comets," The Astrophysical Journal, vol. 355, no. 2, pp. 667-679, 1990.

[30] M. E. Bailey, J. E. Chambers, and G. Hahn, "Origin of sungrazers: a frequent cometary end-state," Astronomy \& Astrophysics, vol. 257, pp. 315-322, 1992.

[31] P. Michel and F. Thomas, "The Kozai resonance for near-Earth asteroids with semimajor axis smaller than 2 AU," Astronomy \& Astrophysics, vol. 307, pp. 310-318, 1996.

[32] F. Thomas and A. Morbidelli, "The Kozai resonance in the outer solar system and the dynamics of long-period comets," Celestial Mechanics \& Dynamical Astronomy, vol. 64, no. 3, pp. 209-229, 1996.

[33] X.-S. Wan and T.-Y. Huang, "An exploration of the Kozai resonance in the Kuiper Belt," Monthly Notices of the Royal Astronomical Society, vol. 377, no. 1, pp. 133-141, 2007.

[34] N. D. Moiseev, "On some basic simplified schemes of celestial mechanics, obtained by means of averaging of restricted circular problem of three bodies 2. On averaged variants of restricted circular three bodies of the spatial bodies," Publication of the State Institute of Astronomy Named after P. K. Sternberg, vol. 15, no. 1, pp. 100-117, Moscow State University named after 
Lomonosov M. V., Scientific Memoirs, Release 96, Originally in Russian, 1945.

[35] K. Ohtsuka, T. Sekiguchi, D. Kinoshita et al., "Apollo asteroid 2005 UD: split nucleus of (3200) Phaethon?" Astronomy \& Astrophysics, vol. 450, no. 3, pp. L25-L28, 2006.

[36] K. Ohtsuka, H. Arakida, T. Ito et al., "Apollo asteroids 1566 Icarus and 2007 MK6: Icarus family members?" The Astrophysical Journal, vol. 668, no. 1, pp. L71-L74, 2007.

[37] P. B. Babadzhanov, G. I. Kokhirova, and Yu. V. Obrubov, "The potentially hazardous asteroid 2007CA19 as the parent of the $\eta$-Virginids meteoroid stream," Astronomy \& Astrophysics, vol. 579, article A119, 2015.

[38] P. B. Babadzhanov and Yu. V. Obrubov, "Evolution of short period meteoroid streams," Celestial Mechanics \& Dynamical Astronomy, vol. 54, no. 1, pp. 111-127, 1992.

[39] G. F. Gronchi and A. Milani, "The stable Kozai state for asteroids and comets: with arbitrary semimajor axis and inclination," Astronomy \& Astrophysics, vol. 341, no. 3, pp. 928-935, 1999.

[40] P. Farinella, L. Foschini, Ch. Froeschlé et al., "Probable asteroidal origin of the Tunguska Cosmic Body," Astronomy \& Astrophysics, vol. 377, no. 3, pp. 1081-1097, 2001.

[41] R. McGehee, "von Zeipel's theorem on singularities in celestial mechanics," Expositiones Mathematicae, vol. 4, no. 4, pp. 335345,1986

[42] G. F. Gronchi and A. Milani, "Averaging on Earth-crossing orbits," Celestial Mechanics \& Dynamical Astronomy, vol. 71, no. 2, pp. 109-136, 1998.

[43] H. Beust, "Orbital clustering of distant Kuiper belt objects by hypothetical Planet 9. Secular or resonant?" Astronomy \& Astrophysics, vol. 590, article L2, 2016.

[44] H. F. Levison and M. J. Duncan, "The long-term dynamical behavior of short-period comets," Icarus, vol. 108, no. 1, pp. 1836, 1994.

[45] J. Wisdom and M. Holman, "Symplectic maps for the $n$-body problem: stability analysis," The Astronomical Journal, vol. 104, no. 5, pp. 2022-2029, 1992.

[46] R. G. Strom, R. Malhotra, T. Ito, F. Yoshida, and D. A. Kring, "The origin of planetary impactors in the inner solar system," Science, vol. 309, no. 5742, pp. 1847-1850, 2005.

[47] T. Ito and R. Malhotra, "Asymmetric impacts of near-Earth asteroids on the Moon," Astronomy \& Astrophysics, vol. 519, article A63, 2010.

[48] T. Ito and S. M. Miyama, "An estimation of upper limit masses of $v$ Andromedae planets," The Astrophysical Journal, vol. 552, no. 1, pp. 372-379, 2001.

[49] T. Ito and K. Tanikawa, "Long-term integrations and stability of planetary orbits in our solar system," Monthly Notices of the Royal Astronomical Society, vol. 336, no. 2, pp. 483-500, 2002.

[50] G. M. Clemence, "The system of astronomical constants," Annual Review of Astronomy and Astrophysics, vol. 3, no. 1, pp. 93-112, 1965.

[51] Y. Kozai, "Secular perturbations of resonant asteroids," Celestial Mechanics \& Dynamical Astronomy, vol. 36, no. 1, pp. 47-69, 1985.

[52] M. Yoshikawa, "A survey of the motions of asteroids in the commensurabilities with Jupiter," Astronomy \& Astrophysics, vol. 213, pp. 436-458, 1989.

[53] M. Moons, "Review of the dynamics in the Kirkwood gaps," Celestial Mechanics \& Dynamical Astronomy, vol. 65, no. 1-2, pp. 175-204, 1996.
[54] G. E. O. Giacaglia, "Secular motion of resonant asteroids", SAO Special Report 278, Smithsonian Institution, Astrophysical Observatory, Cambridge, Mass, USA, 1968.

[55] G. E. O. Giacaglia, "Resonance in the restricted problem of three bodies," The Astronomical Journal, vol. 74, pp. 1254-1261, 1969.

[56] M. Saillenfest, M. Fouchard, G. Tommei, and G. B. Valsecchi, "Long-term dynamics beyond Neptune: secular models to study the regular motions," Celestial Mechanics \& Dynamical Astronomy, vol. 126, no. 4, pp. 369-403, 2016.

[57] T. Gallardo, "The occurrence of high-order resonances and Kozai mechanism in the scattered disk," Icarus, vol. 181, no. 1, pp. 205-217, 2006.

[58] S. J. Kortenkamp, R. Malhotra, and T. Michtchenko, "Survival of Trojan-type companions of Neptune during primordial planet migration," Icarus, vol. 167, no. 2, pp. 347-359, 2004.

[59] T. Gallardo, "Atlas of the mean motion resonances in the Solar System," Icarus, vol. 184, no. 1, pp. 29-38, 2006.

[60] N. D. Moiseev, "On some basic simplified schemes of celestial mechanics, obtained by means of averaging of restricted circular problem of three bodies 1 . On averaged variants of restricted circular three bodies of the plane problem," Publication of the State Institute of Astronomy named after P. K. Sternberg, vol. 15, no. 1, pp. 75-99, Moscow State University named after Lomonosov M. V., Scientific Memoirs, Release 96. Originally in Russian, 1945.

[61] Y. F. Gordeeva, "Time-dependence of orbital elements in longperiod oscillations in the three-body boundary-value problem," Cosmic Research, vol. 6, pp. 450-453, 1968.

[62] M. A. Vashkov'yak, "Evolution of the orbits of distant satellites of Uranus," Astronomy Letters, vol. 25, no. 7, pp. 476-481, 1999.

[63] L. Luo, B. Katz, and S. Dong, "Double-averaging can fail to characterize the long-term evolution of Lidov-Kozai Cycles and derivation of an analytical correction," Monthly Notices of the Royal Astronomical Society, vol. 458, no. 3, pp. 3060-3074, 2016. 

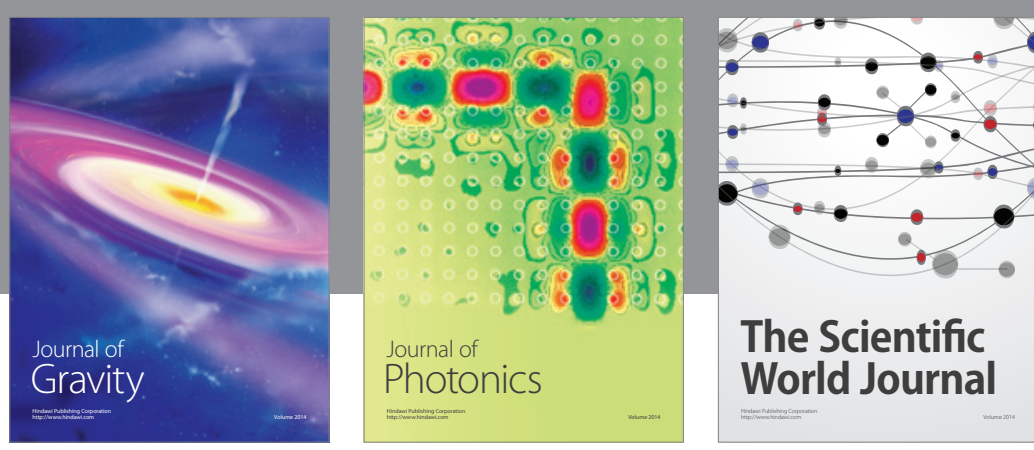

The Scientific World Journal
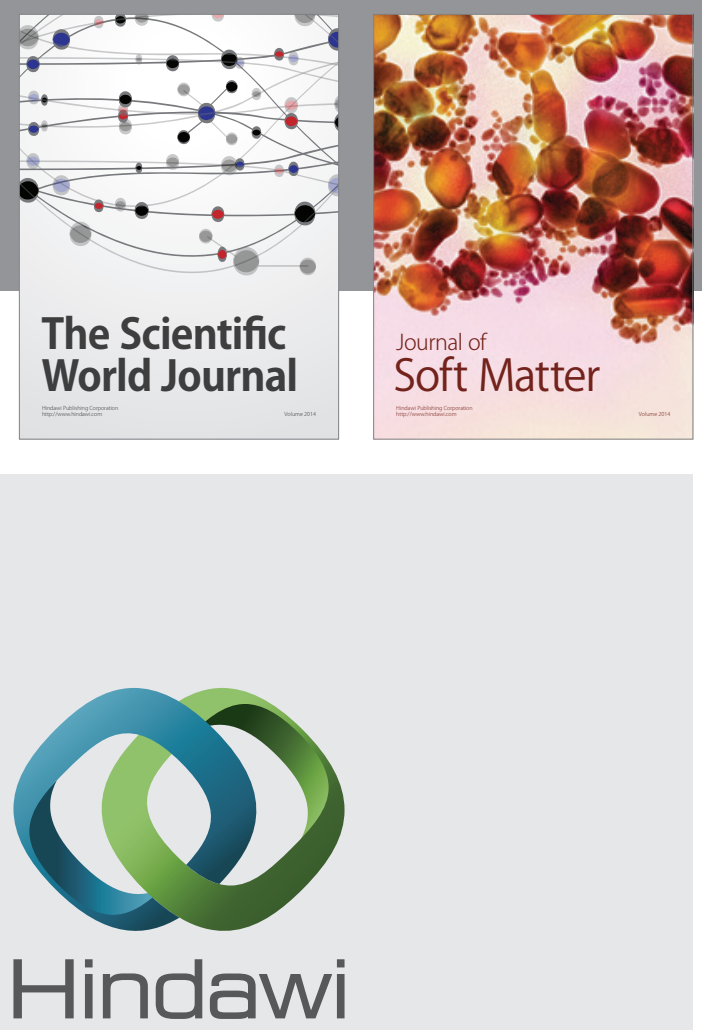

Submit your manuscripts at

http://www.hindawi.com

nternational Journal of

Statistical Mechanics
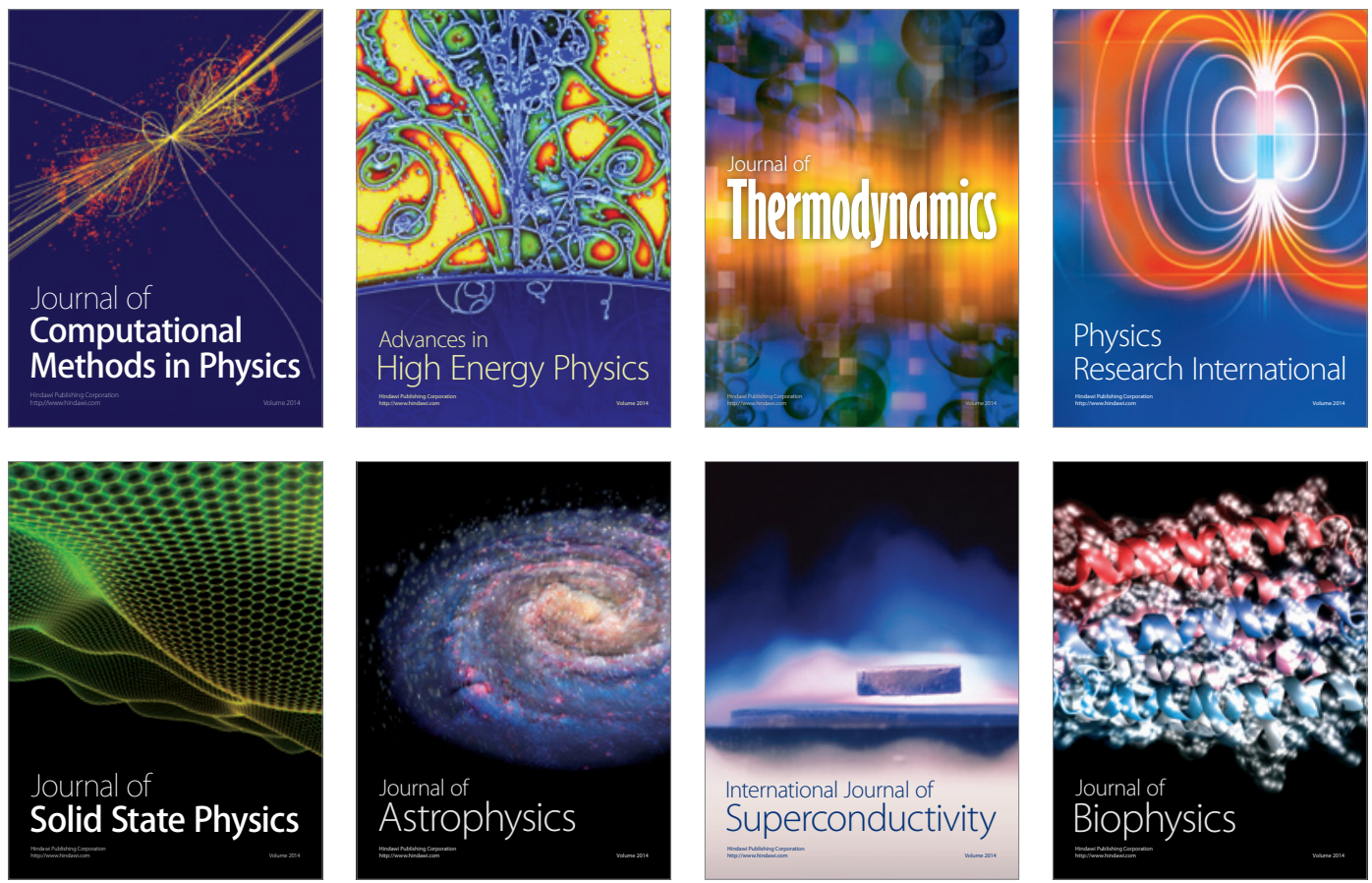
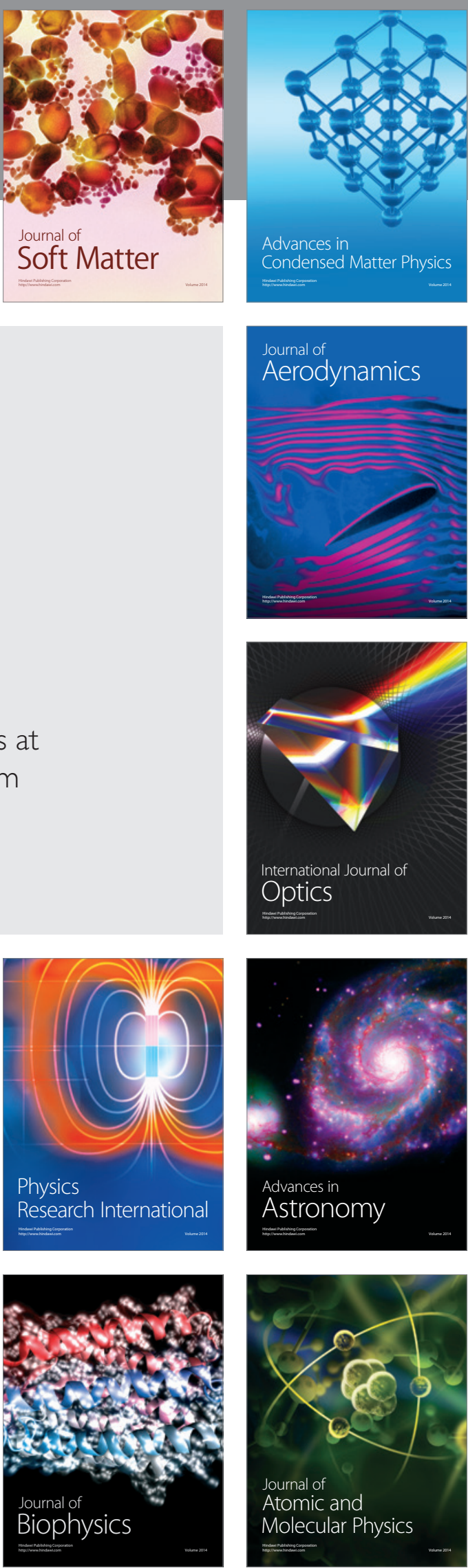